\title{
THE
}

1986

\section{Dynamics of Potential Vorticity Fronts and Eddy Detachment}

\author{
L. J. Pratt \\ University of Rhode Island \\ Melvin E. Stern \\ University of Rhode Island
}

Follow this and additional works at: https://digitalcommons.uri.edu/gsofacpubs

\section{Citation/Publisher Attribution}

Pratt, L. J., \& Stern, M. E. (1986). Dynamics of Potential Vorticity Fronts and Eddy Detachment. J. Phys. Oceanogr., 16(6), 1101-1120. doi: 10.1175/1520-0485(1986)0162.0.C0;2

Available at: https://doi.org/10.1175/1520-0485(1986)0162.0.C0;2

This Article is brought to you for free and open access by the Graduate School of Oceanography at DigitalCommons@URI. It has been accepted for inclusion in Graduate School of Oceanography Faculty Publications by an authorized administrator of DigitalCommons@URI. For more information, please contact digitalcommons-group@uri.edu. 


\title{
Dynamics of Potential Vorticity Fronts and Eddy Detachment
}

\author{
L. J. Pratt* AND Melvin E. STERN \\ Graduate School of Oceanography, University of Rhode Island, Kingston, RI 02881
}

(Manuscript received 11 September 1985, in final form 27 December 1985)

\begin{abstract}
The formation and detachment of quasi-geostrophic eddies in a $1 \frac{1 / 2}{2}$ layer jet is studied using a piecewise uniform potential vorticity model. A vorticity front separates the two pieces, and thus the jet has a cusplike character. The evolution of large amplitude initial disturbances (whose origin may be attributed to barotropicbaroclinic instability mechanisms not explicit in our model) is computed by the method of contour dynamics. Certain numerical results such as the steepening of the front prior to eddy detachment can be physically explained in terms of differential mean field advection and vortex induction. Computations are made for a variety of initial conditions and we indicate the amplitude/scale conditions necessary for the detachment of an eddy. The discussion is directed to the problem of the formation of warm/cold rings in the Gulf Stream. The effect of a coast on large perturbations of a jet is also briefly discussed.
\end{abstract}

\section{Introduction}

Recent advances in remote sensing technology have led to a greater appreciation of the rich mesoscale eddy structure of the Gulf Stream System. Our work centers on the intense rings and ringlike eddies of deformation radius scale which are formed when a segment of the Stream becomes detached from its main axis. Figures 1 and 2 (redrawn from Weatherly et al., 1984, and from Weatherly and Kelly, 1985) show several such events as illustrated by the NWS/NOAA surface frontal maps of the Stream near $40^{\circ} \mathrm{N}, 62^{\circ} \mathrm{W}$. Figure 1a contains a time sequence of maps from July and August 1981 , showing the "pinching off" of a warm core eddy. On day $7 / 13$ the surface front executes a northward, anvil-shaped meander. A week later (day 7/20) we see a cold tongue of fluid (see arrow) wrapping around the anvil from the west and a similar tongue approaches from the east on day $7 / 27$. By day $8 / 3$ the meander remains attached by only a thin thread of warm fluid. A similar sequence of events during May and June 1983 is shown in Fig. 1b. Here, detachment of the northward meander is effected by a western tongue alone.

There are also many cases in which large and steep meanders of the Stream resist detachment for long periods of time. Figure 2 shows a southward meander of the Stream over a two-month period in 1982. Although pinching-off appears imminent on days $5 / 30$ and $6 / 3$, the meander widens (day 6/7) and persists until day $7 / 19$ at which time a blob of cold fluid apparently becomes detached from the southern extremity. This se-

* Present address: Scripps Institution of Oceanography/A-030, La Jolla, CA 92093. quence suggests the presence of some mechanism which works against the pinching off process.

Detached eddies have been reproduced in a number of quasi-geostrophic, numerical models (e.g., Rhines, 1976; Semtner and Mintz, 1977; McWilliams et al., 1978). To date, the most focused numerical studies of eddy detachment are those of Ikeda (1981) and Ikeda and Apel (1981) who used a two-layer, quasi-geostrophic model to study the growth of small disturbances on an eastward jet. For periodic disturbances, Ikeda (1981) finds that detached eddies can occur as the result of meander growth due to baroclinic instability. Once large, the southern and northern extremities of the meander develop anomalous cyclonic and anticyclonic vorticity due to their movement within the planetary potential vorticity gradient. Exterior fluid is wrapped around these extremities and detachment occurs. Baroclinic instability and a (weak) planetary potential vorticity gradient appear to be essential to the whole process. For isolated disturbances Ikeda and Apel (1981) also find detached eddies, but here the detachment is assisted by two mechanisms not observed in the periodic case. The first mechanism involves interactions between neighboring meanders and occurs when a fast, small amplitude meander overtakes a slow, large amplitude meander. This interaction appears to assist detachment of fluid associated with the larger meander, but the physics of the situation is unclear. The second mechanism involves the generation of broad recirculations to the north and south of the jet. Once established, these recirculations are able to cut off the tips of intruding meanders through simple advection.

The purpose of this paper is to elucidate the physics of the "pinching off" process whereby a segment of 

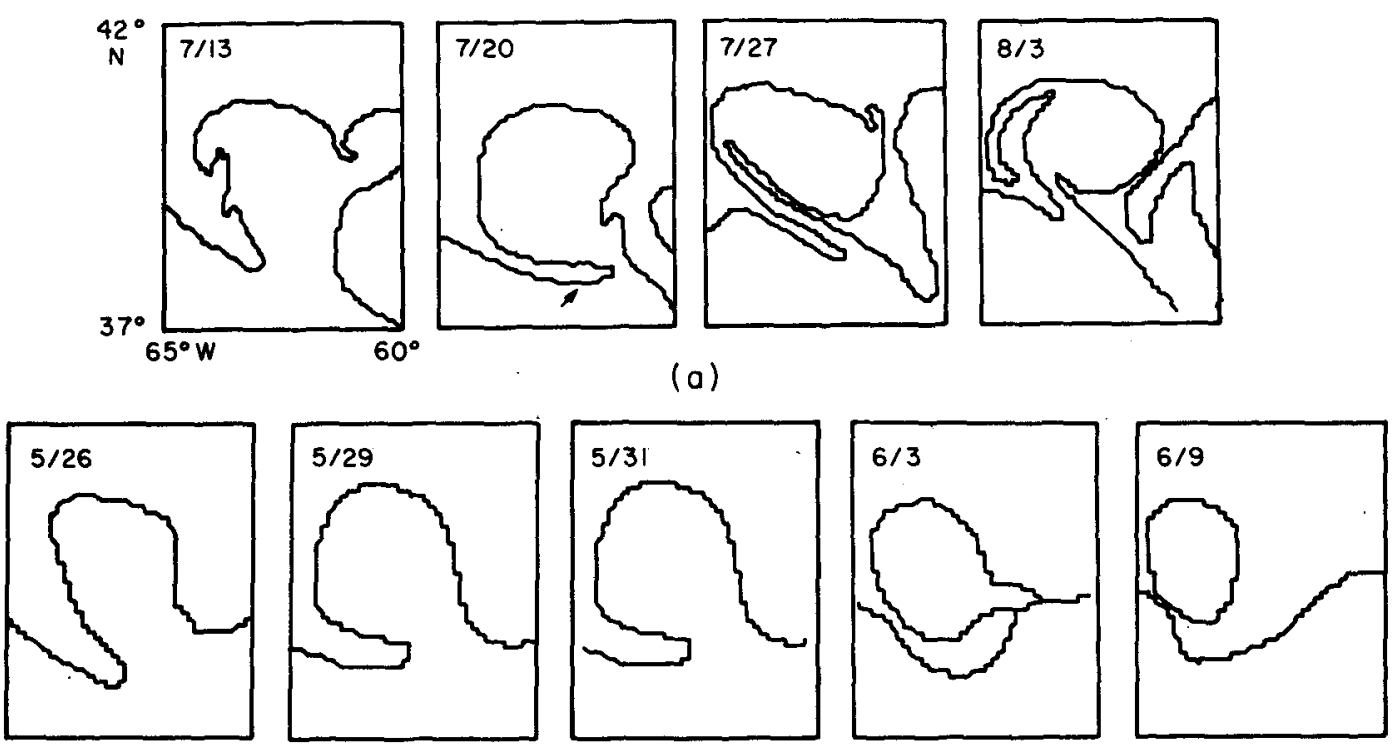

(b)

FIG. 1. Surface frontal positions of the Gulf Stream near $62^{\circ} \mathrm{W}$. (a) 3-week period in 1981 showing detaching warm eddy; (b) 2-week period in 1983 showing detaching warm eddy.

the Stream detaches to form a closed circulation. To do so, we introduce an approach based on the method of contour dynamics (Zabusky and Overman, 1981) in which the location as a function of time of a material contour is the dependent variable. This method is applied to a quasi-geostrophic model containing piecewise uniform potential vorticity. The contour in question is a potential vorticity front centered on a cusped jet. Our study attempts to isolate the conditions under which segments of the front pinch off and form closed contours.

We recognize that a baroclinic/barotropic instability mechanism and possibly others are responsible for the initiation of such finite amplitude disturbances as are shown in Figs. 1 and 2. But important inertial effects come into play when the disturbance amplitudes become finite, and these may be independent of the instability process, particularly if saturation occurs at
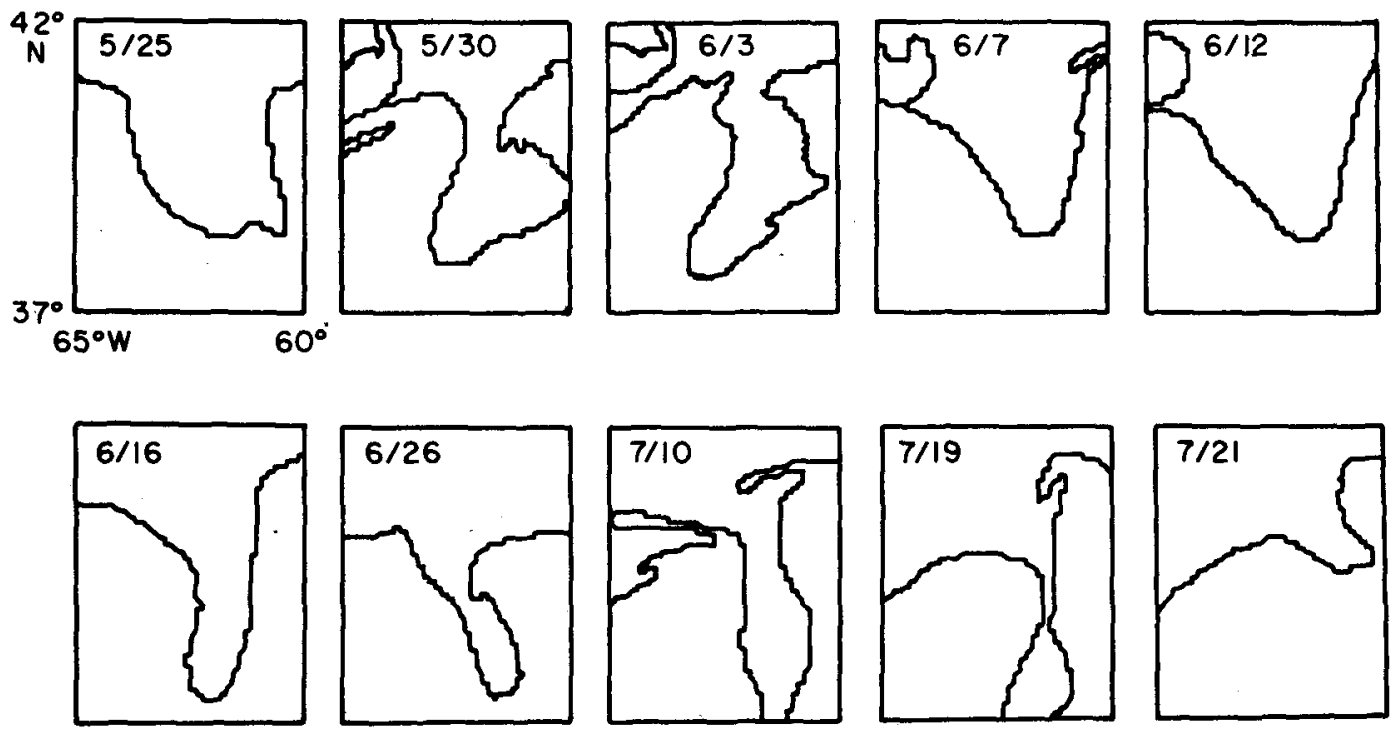

FIG. 2. Same location as in Fig. 1. Here the surface front executes a persistent meander over the two-month period in 1982. Apparently a cold-core eddy finally detaches on about day $7 / 19$. 
moderate amplitude. In trying to isolate the detachment mechanism and to simplify the overall problem we therefore concentrate on following given finite initial disturbances of an otherwise stable jet and try to identify those physical processes which assist and those which work against pinching off. The goal is to determine the extent to which several simple inertial mechanisms can account for the striking observed features mentioned earlier.

\section{The model}

Recent direct measurements of horizontal velocity in the upper levels of the Gulf Stream east of Cape Hatteras have enabled Hall (1985) to reconstruct a potential vorticity section across the Stream. Figure 3, redrawn from her Fig. 4.2, shows potential vorticity along the $14^{\circ} \mathrm{C}$ isotherm across the Stream at $68^{\circ} \mathrm{W}$. The most notable feature of this profile is the steep northward gradient or "wall" centered near $y=0$. The maximum value of this gradient is approximately 60 times the planetary potential vorticity gradient (indicated by the slope of the dashed line in Fig. 3). Similar profiles have been found in eddy-resolving Gulf Stream spin-up models. In Fig. 4 we have reproduced an example from a 3-layer model (Hall, 1985) showing upper layer quasi-geostrophic potential vorticity. As before, the main feature of the profile is a steep wall near $y$ $=0$ separating high potential vorticity (north) from low potential vorticity (south).

Now consider a simple model of the upper Gulf Stream in which the horizontal potential vorticity gradient is completely concentrated in a front and, moreover, all motion is confined to a single density layer overlying a deep inactive layer of slightly lower density. The motion of the upper layer is then equivalent to

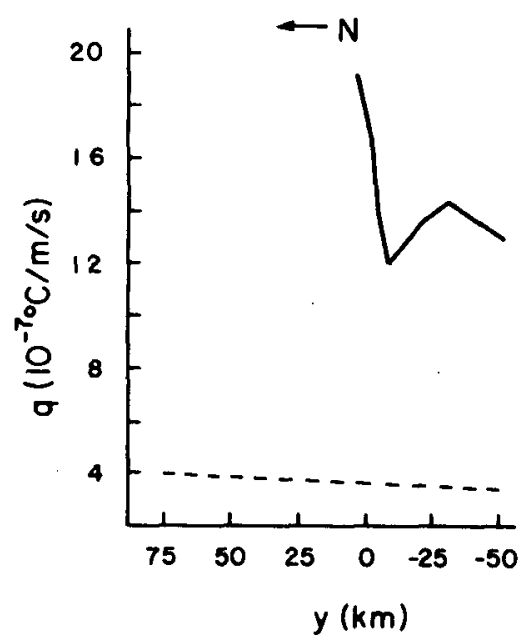

FIG. 3. Mean Ertel potential vorticity along the $14^{\circ} \mathrm{C}$ isotherm along a cross-Gulf Stream section near $68^{\circ} \mathrm{W}$. The slope of the dashed line indicates the planetary potential vorticity gradient. (From Hall, 1985.)

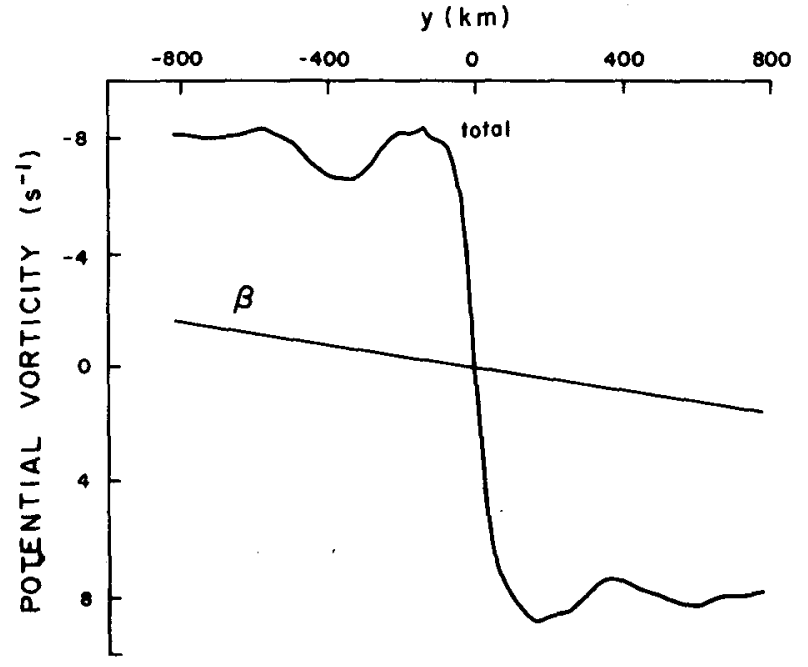

FIG. 4. A north-south section of mean quasi-geostrophic potential vorticity within the upper layer of Holland's 3-layer general circulation model of the Gulf Stream system. (From Hall, 1985.)

that of a single layer under reduced gravity. Such a model might be the first in a hierarchy of models in which uniform potential vorticity and density regions are pieced together to build up a realistic Gulf Stream. As sketched in Fig. 5, the front has horizontal position $y=L(x, t)$ and separates regions of uniform potential vorticities $A$ (for $y>L$ ) and $B-A$ (for $y<L$ ). In accordance with the observed potential vorticity, we will call positive $y$ north, and assume that $A>B-A$, so that the front divides high uniform potential vorticity fluid in the north from low uniform potential vorticity in the south. Although unrelated to the Gulf Stream problem, it is convenient (for later work on coastal jets) to allow for the possibility that a rigid boundary may exist at $y=-L_{0}$.

Let $D$ denote the mean thickness of the upper layer, $d$ a typical cross-stream depth variation of the undisturbed state, $g^{*}$ the reduced gravity, and $f$ the Coriolis parameter. Then if the Rossby radius of deformation $(g * D)^{1 / 2} / f$ is used as the unit of horizontal length, and if the thickness variation $h$ is nondimensionalized using $d$, then the quasi-geostrophic potential vorticity equation for the upper layer implies

$$
\nabla^{2} h-h=\left\{\begin{array}{l}
A, \quad y>L \\
B-A, \quad y<L
\end{array}\right.
$$

where $A$ and $B-A$ denote the potential vorticities nondimensionalized by $f d / D^{2}$. With time $t$ nondimensionalized by $(f d / D)^{-1}$ and velocity by $\left(g^{*}\right)^{1 / 2} d / D^{1 / 2}$ the eastward and northward velocities are given respectively by

$$
\begin{aligned}
& u=-h_{y} \\
& v=h_{x} .
\end{aligned}
$$




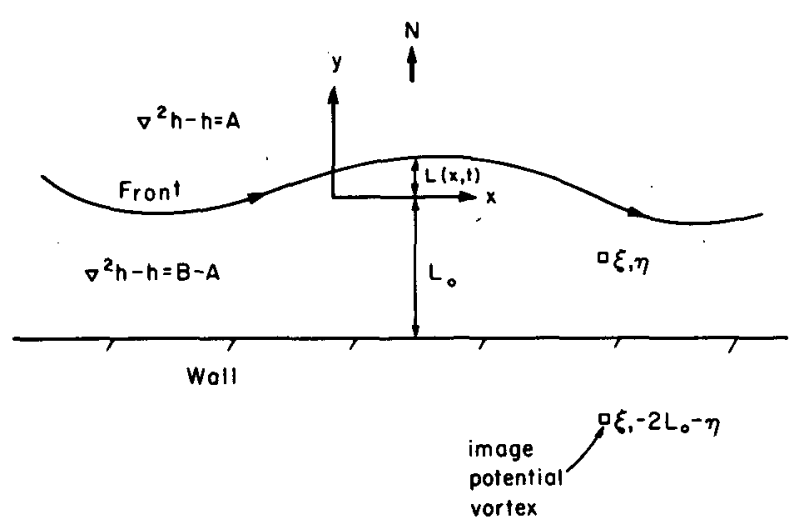

FIG. 5. Definition sketch showing plan view of potential vorticity front. All variables are dimensionless. For the particular case of a free jet remove the wall (i.e., $L_{0}=\infty$ ).

These quasi-geostrophic equations are valid for $d / D$ $\ll 1$ provided the parcel accelerations do not become infinite in the course of time.

The velocities are continuous across the vorticity front, and for the general problem posed in Fig. 5 we will need the condition of no normal flow at the rigid boundary. The geostrophic relation then implies

$$
h_{x}\left(x,-L_{0}, t\right)=0 \text {. }
$$

If $L=0$ is taken as the undisturbed position of the front, then the undisturbed thickness obtained from $(2.1)$ is

$$
h_{0}(y)=\left\{\begin{array}{l}
A\left(e^{-y}-1\right), \quad y>0 \\
-A e^{y}+B \cosh y+A-B, \quad y<0,
\end{array}\right.
$$

and the associated undisturbed velocity is

$$
u_{0}(y)=A e^{-|y|}-\left\{\begin{array}{l}
0, \quad y>0 \\
B \sinh y, \quad y<0 .
\end{array}\right.
$$

This basic flow can describe two different types of jets: one having $B=0$ and a maximum velocity $A$ at the front; and the second having $A=0$ and a maximum velocity $B \sinh \left(L_{0}\right)$ at the wall. The Gulf Stream jet corresponds to the former with $L_{0}=\infty$. The use of the term "jet" must be qualified by noting that conventional jets (such as the ones considered by Ikeda, 1981, and Ikeda and Apel, 1981) are linearly unstable, whereas the "cusped jet" considered here is linearly stable.

The total streamfunction will be represented as a sum of $h_{0}$ and a time-dependent deviation $h^{\prime}$ :

$$
h(x, y, t)=h_{0}(y)+h^{\prime}(x, y, t) .
$$

Using (2.1) and (2.3) then gives

$$
\nabla^{2} h^{\prime}-h^{\prime}= \begin{cases}B-2 A, & L>y>0 \\ 2 A-B, & L<y<0 \\ 0, & \text { otherwise. }\end{cases}
$$

The $B-2 A$ and $2 A-B$ terms on the right-hand side of (2.6) represent the difference between the potential vorticity underneath a ridge $(L>y>0)$ or trough $(L<y<0)$ and the basic potential vorticity. Hence, the $h^{\prime}$ component is appropriately referred to as the vortex anomaly component. The separation of the total flow into an $h^{\prime}$ component (which must vanish at $y$ $=-\infty$ ) and a mean field component will provide us with a good deal of physical insight into the numerical solutions.

The solution to (2.6) can be constructed using the Greens kernel for the modified Helmholtz operator $\left(\nabla^{2}-1\right)$ :

$$
G(x, y, \xi, \eta)=\frac{1}{2 \pi} K_{0}\left\{\left[(x-\xi)^{2}+(y-\eta)^{2}\right]\right\}^{1 / 2}
$$

where $\xi$ and $\eta$ denote the $x$ - and $y$-coordinates of a point source of potential vorticity and $K_{0}(z)$ is the modified Bessel function of zero-order. Integrating the product of (2.7) and the right-hand side of (2.6) over all $\xi$ and $\eta$ gives

$$
\begin{aligned}
h^{\prime}(x, y, t)= & \int_{-\infty}^{\infty} d \xi \int_{-\infty}^{\infty} d \eta\left\{\begin{array}{r}
B-2 A,(L>\eta>0) \\
2 A-B,(L<\eta<0) \\
0,
\end{array}\right\} \\
& \times\left\{G(x, y ; \xi, \eta)-G\left(x, y ; \xi,-\eta-2 L_{0}\right)\right\} \\
= & \int_{-\infty}^{\infty} d \xi\left\{\begin{array}{l}
\int_{0}^{L(\xi, t)} d \eta(B-2 A), L(\xi, t)>0 \\
\int_{L(\xi, t)}^{0} d \eta(2 A-B), L(\xi, t)<0
\end{array}\right\} \\
& \times\left\{G(x, y ; \xi, \eta)-G\left(x, y ; \xi,-\eta-2 L_{0}\right)\right\} \\
= & (B-2 A) \int_{-\infty}^{\infty} d \xi \int_{0}^{L(\xi, t)} d \eta\{G(x, y ; \xi, \eta) \\
& \left.-G\left(x, y ; \xi,-\eta-2 L_{0}\right)\right\} . \quad(2.8)
\end{aligned}
$$

The second Bessel function on the right-hand side of (2.8) represents the image point required to satisfy the wall condition (2.2).

The time-dependent horizontal velocities are given by

$$
\begin{array}{r}
u^{\prime}(x, y, t)=-h_{y}^{\prime}=(B-2 A) \int_{-\infty}^{\infty} d \xi \int_{0}^{L(\xi, t)} d \eta \partial / \partial \eta \\
\quad \times\left\{G(x, y ; \xi, \eta)+G\left(x, y ; \xi,-\eta-2 L_{0}\right)\right\} \\
=(B-2 A) \int_{-\infty}^{\infty} d \xi\{G[x, y ; \xi, L(\xi, t)]-G(x, y ; \xi, 0) \\
\left.\left.\quad+G\left[x, y ; \xi,-L(\xi, t)-2 L_{0}\right)\right]-G\left(x, y ; \xi,-2 L_{0}\right)\right\}
\end{array}
$$




$$
\begin{aligned}
v(x, y, t)=h_{x}^{\prime} & =(B-2 A) \partial / \partial x \int_{-\infty}^{\infty} d \xi \int_{0}^{L(\xi, t)} d \eta \\
& \times\left\{G(x, y ; \xi, \eta)-G\left(x, y ; \xi,-\eta-2 L_{0}\right)\right\} \\
=(2 A-B) & \int_{-\infty}^{\infty} d \alpha \partial / \partial x \int_{0}^{L(x-\alpha, t)} d \eta \\
\times & \left\{G(0, y ; \alpha, \eta)-G\left(0, y ; \alpha,-\eta-2 L_{0}\right)\right\} \\
=(B-2 A) & \int_{L(-\infty, t)}^{L(\infty, t)} d L\{G[x, y ; \xi, L(\xi, t)] \\
& \left.\quad-G\left[x, y ; \xi,-L(\xi, t)-2 L_{0}\right]\right\} . \quad(2.9 \mathrm{~b}) .
\end{aligned}
$$

In the last expression, it is understood that the integration with respect to $d L$ covers all values of $L$ on the interval $-\infty<\xi<\infty$. These equations are valid even where $L$ is a multivalued function of $x$ (Stern, 1985), in which case the integrals are line integrals.

\section{Calculation of $L(x, t)$ : Contour dynamics}

We now focus on the problem of determining $L(x, t)$ given $L(x, 0)$. Consider the motion of a tagged frontal parcel having position $[x(t), L(t)]$. The $x$-velocity of the parcel $d x / d t=u_{0}(L)+u^{\prime}(x, L, t)$ is obtained by evaluating (2.9a) at $y=L(x, t)$ and adding the result to (2.4) evaluated at $y=L$. The $y$-velocity of the parcel $d L / d t=v(L)$ is due entirely to anomalous vorticity. A form of these equations obtained by carrying out two of the integrations in (2.9a) is

$$
\begin{aligned}
d x / d t= & (B-2 A) \int_{-\infty}^{\infty} d \xi\{G[x, L(x, t) ; \xi, L(\xi, t)] \\
+ & \left.G\left[x, L(x, t) ; \xi,-L(\xi, t)-2 L_{0}\right]\right\} \\
- & \frac{(2 A-B)}{2}\left[e^{-|L(x, t)|}+e^{-\left\{L(x, t)+2 L_{0}\right\}}\right] \\
+ & A e^{-|L(x, t)|}-\left\{\begin{array}{ll}
0, & L>0 \\
B \sinh L(x, t), & L<0
\end{array}\right\} \quad(3.1 \mathrm{a}) \\
d L / d t= & (B-2 A) \int_{L(-\infty, t)}^{L(\infty, t)} d L\{G[x, L(x, t) ; \xi, L(\xi, t)] \\
& \left.-G\left[x, L(x, t) ; \xi,-L(\xi, t)-2 L_{0}\right]\right\} . \quad(3.1 \mathrm{~b})
\end{aligned}
$$

The relation

$$
\int_{-\infty}^{\infty} d z K_{0}\left[(a-z)^{2}+b^{2}\right]^{1 / 2}=\pi e^{-|b|}
$$

(see the Fourier transforms on page 118 of Magnus and Oberhettinger) has been used to perform several integrations in (3.1a).

For our main problem in which $L_{0} \rightarrow \infty, B \rightarrow 0$ we may set $A=1$ without loss of generality (this sets the $h$ metric equal to half the difference of the dimensional thickness values at $\left.y^{\prime}= \pm \infty\right)$, whereupon $(3.1 \mathrm{a}, \mathrm{b})$ reduce to

$$
\begin{aligned}
& d x / d t=-2 \int_{-\infty}^{\infty} d \xi G[x, L(x, t) ; \xi, L(\xi, t)] \\
& d L / d t=-2 \int_{L(-\infty, t)}^{L(\infty, t)} d L G[x, L(x, t) ; \xi, L(\xi, t)] .
\end{aligned}
$$

An alternate form of (3.3a) obtained using (3.2) is

$$
\begin{aligned}
d x / d t=e^{-|L(x, t)|}-2 \int_{-\infty}^{\infty} & d \xi\{G[x, L(x, t) ; \xi, L(\xi, t)] \\
& -G[x, L(x, t) ; \xi, 0]\}
\end{aligned}
$$

In this form the partition of the $x$-velocity into basic flow plus vortex anomaly contributions is explicit.

In principle equations $(3.3 \mathrm{a}, \mathrm{b})$ determine the " $\mathrm{dy}$ namics" of the contour $y=L(x, t)$ given $L(x, 0)$. However, closed form solutions are difficult without further approximation due to the complicated structure of the integrands. Instead, consider a numerical solution based on a Lagrangian scheme in which the front is resolved into $n$ material parcels with positions $x_{i}(t)$ and $L_{i}(t), i$ increasing monotonically with arclength along the front. The velocity of each parcel is found using trapezoidal approximations to evaluate the integrals in (3.1), (3.3) or (3.4) and a predictor-corrector method is used to implement time-stepping. This algorithm forms the basis for all calculations presented in sections 5 and 6 and is described in detail in the Appendix. The essentially Lagrangian calculation allows $L(x, t)$ to become a multivalued function of $x$ without necessitating any special computation. However, the algorithm does break down if grazing contact is made between different pieces of the front, as occurs in the neck of a detaching eddy. Although it is questionable whether mathematically conclusive contact can occur in the nondiffusive equations under consideration, the neck width can become arbitrarily small and contact may occur due to numerical error. When very small neck widths are observed, the numerical integration is stopped, the physical justification being that close contact implies an important intervention of small-scale processes (not included in the quasi-geostrophic framework) which will complete the detachment process.

The method of contour dynamics has been used to study the evolution of various types of barotropic vortices (e.g., Zabusky et al., 1979; Zubusky and Overman, 1981; Overman and Zabusky, 1982). More pertinent to the present problem are studies by Pullin (1981) and Stern and Pratt (1985) of a two-dimensional front separating regions of irrotational and finite uniform vorticity fluid. Here, wave breaking and folding of the front followed by entrainment of irrotational fluid by the finite vorticity fluid is observed for sufficiently large or steep initial disturbances. However, captured blobs of irrotational fluid are stretched into long filaments by 
the surrounding vortical fluid and are thus unable to exert any significant dynamical influence. Stern (1985) explored the equivalent barotropic version of this problem by considering the case $\left(L_{0}=\infty, A=0\right.$, $B=2$ ) of Eq. (2.1). Here the basic state is motionless to the north of the front and is cyclonically sheared to the south. Sufficiently large meanders tend to steepen, causing large-scale lateral wave breaking resembling shingle-shaped disturbances in the Gulf Stream. Also, low vorticity coastal water is engulfed in the cyclonic shear zone and then stretched out to the point of dynamical insignificance. We emphasize this because the behavior is remarkably different from the detachment to be discussed. Before doing this it is convenient to list some elementary facts and some limiting cases of the Lagrangian integro-differential equations for $(L, x)$.

\section{Limiting and special cases \\ a. Periodic disturbances of small amplitude}

It is easy to derive an equation for the propagation speed $c$ of infinitesimal amplitude (a) disturbances of the form $h^{\prime}(x, y, t)=a \cos [k(x-c t)] g(y)$. One substitutes this into the linearized potential vorticity equation, uses the conditions of pressure continuity and mass conservation at the front, and readily obtains:

$$
c=A-\frac{2 A-B}{\gamma\left[1+\operatorname{coth}\left(\gamma L_{0}\right)\right]}
$$

where $\gamma=\left(1+k^{2}\right)^{1 / 2}$. If the front is free $(A=1$, $\left.B=0, L_{0}=\infty\right)$ then $(4.1)$ reduces to

$$
c=1-\left(1+k^{2}\right)^{-1 / 2}
$$

and the corresponding group speed is

$$
c_{g}=d(c k) / d k=1-\left(1+k^{2}\right)^{-3 / 2} .
$$

In both the bounded and free cases, the frontal waves are neutrally stable and propagate with the centerline jet speed minus a speed due to vortex induction. The latter can be described rather easily for the free case by considering the motion of the front near the disturbance sketched in Fig. 6. The lateral (north-south) motion of any fluid parcel at the front is due primarily to anomalous vorticity associated with disturbances of the front within several deformation radii of that parcel.

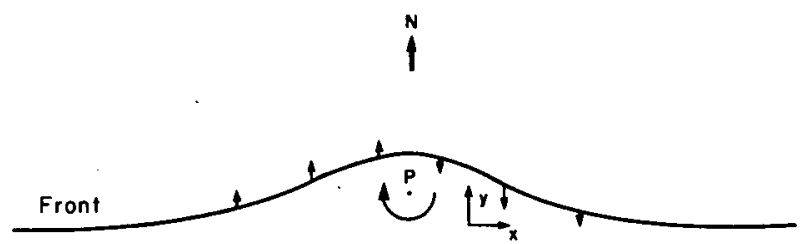

FIG. 6. Illustration of the vortex induction effect on the front near an isolated ridge. The vortex induction is represented by an imaginary point vortex labeled $P$.
In Fig. 6 the disturbance consists of a single ridge which lies to the north of $y=0$ and therefore contains anomalously low (anticyclonic) vorticity. We may qualitatively represent the integrated effect of this anomaly using a point vortex at point $P$. This vortex moves the front southward downstream (to the east) of the crest and northward upstream of the crest, leading to a westward propagation relative to the $u_{0}(0)$. Later on in the dispersive process the southward displacements to the right of the crest will lead to a high vortex anomaly (trough) and eventually more crests and troughs will appear further downstream. When the boundary is present, the phase speed (4.1) can be understood using similar arguments.

\section{b. The long-wave limit}

Next consider disturbances of finite amplitude and wave length large compared to both $L_{0}$ and unity. Formally we write

$$
L(x, t)=L^{(0)}(X, t)+\mathrm{O}(\epsilon)
$$

where $X=\epsilon x$ and where again $\epsilon \ll 1, L_{0}$. Taylor expanding $L(\xi, t)$ about $\xi=x$ then gives

$L(\epsilon \xi, t)-L(\epsilon x, t)=\epsilon(\xi-x) \frac{\partial L(x, t)}{\partial X}+O\left(\epsilon^{2}\right)$.

If (4.3) and (4.4) are used in (3.1), the $x$-velocity at the front becomes

$$
\begin{array}{r}
u(x, L, t)=\frac{2 A-B}{2}\left(1-e^{-|L|}+e^{-2|L+L 0|}-e^{-|L+2 L 0|}\right) \\
+A e^{-|L|}-\left\{\begin{array}{ll}
0, & y>L \\
B \sinh (L), & y<L
\end{array}\right\}+O(\epsilon)
\end{array}
$$

whereas the $y$-velocity becomes

$v(x, L, t)=\frac{2 A-B}{2}\left(1-e^{-2|L+L 0|}\right) \partial L / \partial x+\mathrm{O}(\epsilon)$.

Finally, substitution of (4.5) and (4.6) into the kinematic relation

$$
v(x, L, t)=\partial L / \partial t+u(x, L, t) \partial L / \partial x
$$

yields, to leading order

$$
\frac{\partial L}{\partial t}+C(L) \frac{\partial L}{\partial x}=0
$$

where the long wave speed $C(L)$ is

$$
\begin{array}{r}
C(L)=\frac{2 A-B}{2}\left(2 e^{-2|L+L 0|}-e^{-|L|}-e^{-|L+2 L 0|}\right) \\
+A e^{-|L|}-\left\{\begin{array}{ll}
0, & (y>L) \\
B \sinh (L), & (y<L)
\end{array}\right\} .
\end{array}
$$

Long finite-amplitude waves steepen according to (4.8) 
as one $L$ catches up to a nearby $L$ having a smaller propagation speed.

When the coastal-trapped portion of the current is absent $(B=0)$ but the boundary remains ( $L_{0}$ is finite), (4.9a) reduces to

$$
C(L)=A\left(2 e^{-2\left|L+L_{0}\right|}-e^{-\left|L+2 L_{0}\right|}\right), \quad B=0
$$

whereas the boundary free case $\left(L_{0} \rightarrow \infty\right)$ yields

$$
C(L)=0, \quad B=0, \quad L_{0}=\infty
$$

i.e., long-wave disturbances of the free front are stationary. Finally the small amplitude, long-wave limit for arbitrary $B, L_{0}$ is

$$
\begin{aligned}
C(L) & =A-\frac{(2 A-B)}{2}\left(1-e^{-2 L_{0}}\right) \\
& =A-\frac{2 A-B}{1+\operatorname{coth}\left(L_{0}\right)}
\end{aligned}
$$

in agreement with the long-wave limit of Eq. (4.1). According to (4.8), each value of $L$ propagates with speed $C(L)$ in the positive $x$ direction. The interesting limit documented in the following subsection for future reference is not used further in this paper, and consequently the reader may prefer to skip to section $4 \mathrm{~d}$.

\section{c. Free disturbances of finite amplitude and small cur- vature}

As demonstrated by (4.9c), long-wave disturbances of the free jet remain stationary to leading order (in deformation radius/wave length), and we now explore the slow evolution of such disturbances as determined by the next terms in the expansion. Let

$$
\begin{aligned}
L(x, t) & =\epsilon^{-1} l(\tau, X)+\mathrm{O}(1) \\
X & =\epsilon x \\
\tau & =\epsilon^{5} t
\end{aligned}
$$

where $\epsilon \ll 1$ and $\zeta$ is a yet undetermined factor determining the slowness of the time scale. Using (3.3a, b) in (4.7) gives

$$
\begin{aligned}
L_{t}=\frac{1}{\pi} \int_{-\infty}^{\infty} & d \xi\left[L_{\xi}(\xi, t)-L_{x}(x, t)\right] \\
& \times K_{0}\left\langle\left\{(x-\xi)^{2}+[L(x, t)-L(\xi, t)]^{2}\right\}^{1 / 2}\right\rangle,
\end{aligned}
$$

and substitution of (4.10) into this expression yields .

$$
\begin{aligned}
\epsilon^{5-1} l_{\tau}= & \frac{1}{\pi} \int_{-\infty}^{\infty} d \xi\left\{\epsilon(\xi-x) l_{X X}+\frac{1}{2} \epsilon^{2}(\xi-x)^{2} l_{X X X}\right\} \\
& \times K_{0}\left\langle\left\{(\xi-x)^{2}+\left[(\xi-x) l_{X}\right.\right.\right. \\
& \left.\left.\left.+\frac{\epsilon}{2}(\xi-x)^{2} l_{X X}+O\left(\epsilon^{2}\right)\right]^{2}\right\}^{1 / 2}\right\rangle
\end{aligned}
$$

where a Taylor expansion in powers of $\xi$ about $\xi=x$ has been used. Letting $z=(\xi-x)$ we get

$$
\begin{aligned}
\pi \epsilon^{\zeta-2} l_{\tau}= & \int_{-\infty}^{\infty} d z z l_{X X} K_{0}\left\{\left[\left(1+l_{X}^{2}\right) z^{2}+\epsilon l_{X X} l_{X} z^{3}\right]^{1 / 2}\right\} \\
& +\frac{\epsilon}{2} \int_{-\infty}^{\infty} d z z^{2} l_{X X X} K_{0}\left\{\left[\left(1+l_{X}^{2}\right) z^{2}\right]^{1 / 2}\right\}+O\left(\epsilon^{2}\right) \\
= & I_{1}+I_{2}+O\left(\epsilon^{2}\right)
\end{aligned}
$$

The integral $I_{1}$ can be evaluated through use of the Bessel function definition:

$$
K_{n}(z)=\int_{0}^{\infty} d \theta \cosh (n \theta) e^{-z \cosh (\theta)} .
$$

If this expression is used in $I_{1}$ and the exponential function is expanded in a Taylor Series about $\epsilon=0$, the result is

$$
\begin{aligned}
& I_{1}= l_{X X} \int_{-\infty}^{\infty} d z z \int_{0}^{\infty} d \theta e^{-\cosh (\theta)\left[z^{2}\left(1+l_{x}\right)\right]^{1 / 2}} \\
&-\frac{\epsilon l_{X X}^{2} l_{X}}{2\left(1+l_{X}^{2}\right)^{1 / 2}} \int_{-\infty}^{\infty} d z \int_{0}^{\infty} d \theta|z|^{3} \\
& \\
& \quad \times \cosh (\theta) e^{-\cosh (\theta)\left[z^{2}\left(1+l_{x}^{2}\right)\right]^{1 / 2}}+O\left(\epsilon^{2}\right) .
\end{aligned}
$$

The first integral vanishes identically since the integrand is odd, leaving $O\left(\epsilon^{2}\right)$

$$
\begin{aligned}
I_{1} & =\frac{-\epsilon l_{X X}^{2} l_{X}}{2\left(1+l_{X}^{2}\right)^{5 / 2}} \int_{-\infty}^{\infty} d \alpha \int_{0}^{\infty} d \theta|\alpha|^{3} \cosh (\theta) e^{-\cosh (\theta)|\alpha|} \\
& =\frac{-\epsilon l_{X X}^{2} l_{X}}{2\left(1+l_{X}^{2}\right)^{5 / 2}} \int_{-\infty}^{\infty} d \alpha|\alpha|^{3} K_{1}(|\alpha|) \\
& =\frac{-3 \epsilon \pi l_{X X}^{2} l_{X}}{2\left(1+l_{X}^{2}\right)^{5 / 2}}
\end{aligned}
$$

The evaluation of the last integral is given by Abramowitz and Stegun (1964). The integral $I_{2}$ can be evaluated in a similar way, and the result is

$$
\begin{aligned}
I_{2} & =\frac{\epsilon l_{X X X}}{\left(1+l_{X}^{2}\right)^{3 / 2}} \int_{0}^{\infty} d \alpha \alpha^{2} K_{0}(\alpha) \\
& =\frac{\epsilon \pi l_{X X X}}{2\left(1+l_{X}^{2}\right)^{3 / 2}}
\end{aligned}
$$

Substitution of (4.13) and (4.14) back into (4.11) yields

$$
\epsilon^{\xi-3} l_{\tau}=\frac{-3 l_{X X}^{2} l_{X}}{2\left(1+l_{X}^{2}\right)^{5 / 2}}+\frac{l_{X X X}}{2\left(1+l_{X}^{2}\right)^{3 / 2}}+O(\epsilon)
$$

so that $\zeta=3$. Therefore, disturbances with (wavelength/ 
deformation radius) $=\epsilon$ will evolve on an $\mathrm{O}\left(\epsilon^{3}\right)$ time scale according to

$$
l_{\tau}-\frac{l_{X X X}}{2\left[1+l_{X}^{2}\right]^{3 / 2}}+\frac{3\left(l_{X X}\right)^{2} l_{X}}{2\left[1+l_{X}^{2}\right]^{5 / 2}}=0 .
$$

Note that the amplitude of $L$ is of the order of the wavelength.

A more intuitive form of (4.15) can be written in terms of the curvature:

$$
\kappa=\frac{l_{X X}}{\left[1+l_{X}^{2}\right]^{3 / 2}} .
$$

The second and third terms in (4.15) constitute $1 / 2$ the $X$-derivative of $\kappa$ so that (4.3) may be rewritten

$$
l_{\tau}-\frac{1}{2} \kappa_{X}=0 \text {. }
$$

This long-wave model is related to but different from earlier thin jet models of the Gulf Stream (e.g., Flierl and Robinson, 1984), and further discussion is reserved for a later paper.

\section{d. Velocity tendency calculations for simple distur- bances}

In order to explain features of the full numerical solutions to Eqs. $(3.3 \mathrm{a}, \mathrm{b})$ which will appear later, we document here the velocities produced by some elementary frontal shapes (e.g., Fig. 7). Consider first an isolated disturbance whose downstream width is much shorter than unity. Since the horizontal area of the fluid bounded by this frontal displacement and the $x$ -

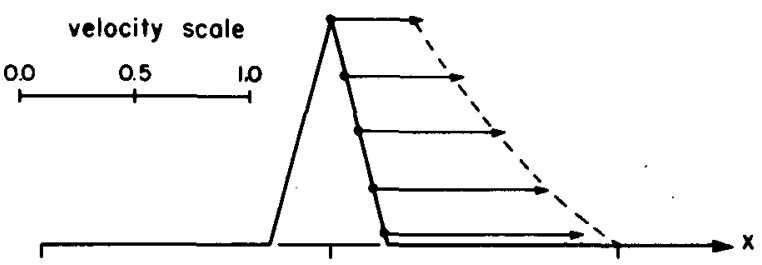

(a)

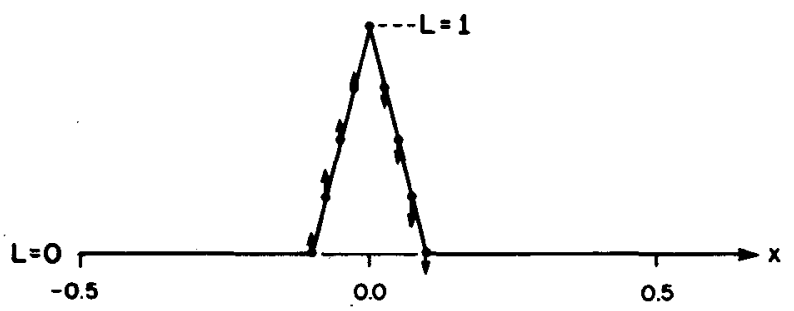

(b)

FIG. 7. Horizontal velocities of various frontal parcels for a spiked $L$ disturbance of free front $(A=1, B=0, L=\infty)$. The dashed line in (a) indicates the $x$-velocity due to the basic flow alone, and the arrows indicate the total velocity. (b) The arrows indicate the $y$-velocity. axis is small, the vortex anomaly is small and therefore the evolution is dominated by the basic velocity (2.4). As an example, we have computed the velocities for the spiked disturbance

$$
L(x, 0)= \begin{cases}1-10|x|, & |x|<0.1 \\ 0, & \text { otherwise }\end{cases}
$$

using Eqs. (3.3a, b). The total $x$-velocities for several material parcels distributed over the downstream (right) face of the spike are indicated by arrows in Fig. 7a, and the $x$-velocities on the upstream face are identical. Compare these with the dashed line which gives the basic flow contribution, and which accounts for most of the $x$-velocity. Also Fig. 7b shows that the $y$-velocities are small compared to the $x$-velocities. It follows that fluid parcels in the southern (i.e., smaller $y$ ) part of the spike travel eastward at a faster rate than those in the northern tip. The rear (west) side of the spike will therefore steepen, tilt backward, and a kind of breaking wave will be produced.

In contrast consider a disturbance having a large area for the potential vortex anomaly, as given by the step:

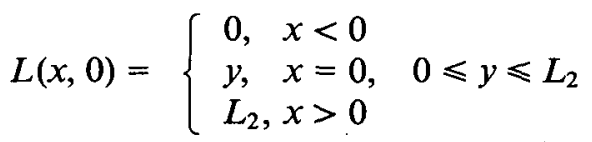

The total horizontal velocity for points on $x=0$ is given by (3.3a) as

$$
\begin{aligned}
u(0, L, 0)= & \frac{1}{\pi} \int_{-\infty}^{0} d \xi K_{0}\left[\left(\xi^{2}+L^{2}\right)^{1 / 2}\right] \\
& +\frac{1}{\pi} \int_{0}^{\infty} d \xi K_{0}\left\{\left[\xi^{2}+\left(L-L_{2}\right)^{2}\right]^{1 / 2}\right\} \\
= & \left(e^{-L}+e^{\left(-L_{2}+L\right)}\right) / 2 .
\end{aligned}
$$

When $L_{2} \gg 1$, the value of (4.17) at the south $(y=0)$ corner is one-half, and near the north corner $\left(y=L_{2}\right)$ the value of (4.17) is also approximately one-half, i.e., the same as at the south corner. The $x$-velocities for the case $L_{2}=1$ are shown in Fig. 8 and indicate the tendencies described above. Specifically, we see that a small amount of frontal folding is confined to the southern part of the step and occurs at a slower rate than that of the spike. The $y$-velocities, given in Fig. $8 \mathrm{~b}$, are now the same magnitude as the $x$-velocities.

It is instructive to compare this tendency calculation to the result obtained by Stern (1985) for a step in a different flow $\left(A=0, B=2, L_{0}=\infty\right)$, which is indicated in the inset on the upper left side of Fig. 8a. Here the basic velocity is zero to the north of $y=0$ so that the east velocity at the step disturbance is entirely due to the (now cyclonic) vortex anomaly under the step. This anomaly tends to tilt the step backward (as shown in Fig. 8a inset) and the upper corner velocity $u\left(L_{2}\right)$ is equal and opposite to that of the lower corner velocity $u(0)$. For our free jet profile, on the other hand, we see 

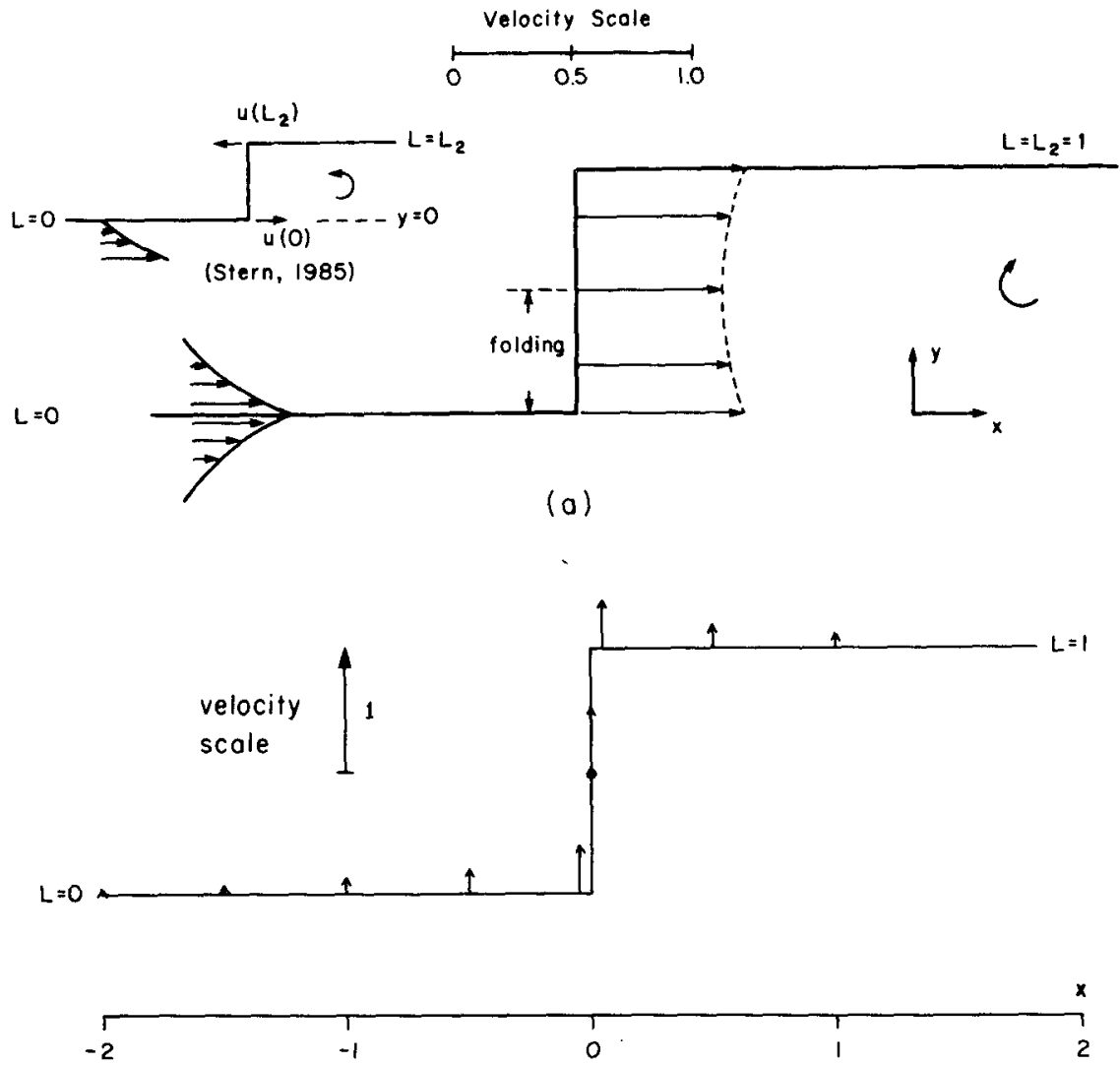

(b)

Fig. 8. The horizontal velocities for a step disturbance of unit amplitude for free case $(A=1$, $B=0, L_{0}=\infty$ ). (a) $u$ along the step. The inset shows a step disturbance of Stern's (1985) front $\left(A=0, B=2, L_{0}=\infty\right)$ with corner velocities. (b) $v$ near the step.

that $u\left(L_{2}\right)=u(0)$ and therefore one should expect the temporal evolution of our step to be much different. The previously discussed spike signature would seem to be the more relevant one for significant frontal steepening and folding.

\section{Numerical results for isolated disturbances of the free front}

We now consider the complete evolution of the front for several classes of initial conditions. For the most part attention is restricted to initial disturbances having downstream widths of the same order of the deformation radius. Longer wave lengths are governed by Eq. (4.15) and will be the subject of a future study. Shorter widths are essentially governed by the barotropic contour dynamics (see Stern, 1985). The vital statistics for all numerical experiments are listed in Table 1 , and an important result is the tabulation of a horizontal area of detaching eddies. This gives some quantitative measure of the pinching off process, such as may be related to the heat flux across the front in the oceanic prototype.

\section{a. The smoothed step}

First consider the initial shape

$$
L(x, 0)=L_{2}\left[1+\tanh \left(S_{0} x\right)\right] / 2
$$

with $L_{2}=4$ and $S_{0}=10$ (labeled $t=0$ in Fig. 9), this being essentially the step profile whose tendency was discussed in the previous section. As anticipated, folding occurs in the southern part of the step (see $t=4$ in Fig. 9) causing $L(x, t)$ to become a multivalued function of $x$. A northward excursion of the front near the step can also be seen and is explained by the tendencies in Fig. 8b. This northward movement carries the step into a region where the basic shear is weaker and the result is that the front "unfolds" $(t=7)$ and the step begins to disperse. By $t=15$ the step has dispersed to a good degree and several trailing waves have developed. This calculation dramatically illustrates the ability of anomalous potential vorticity to disperse disturbances even when huge initial slopes are introduced.

An eastward-facing step evolves in essentially the same way as a westward-facing step of the same dimensions. This follows from the fact that $-L(x, t)$ is 


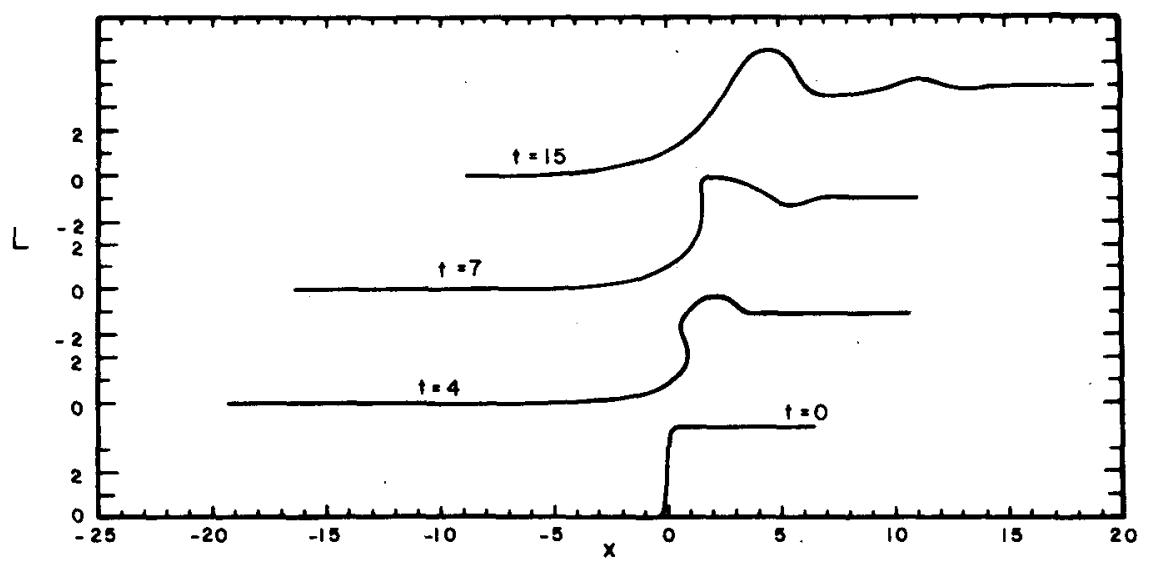

FIG. 9. Evolution of the case $L(x, 0)=2[1+\tanh (10 x)]$.

a solution to the equations of contour dynamics if $L(x, t)$ is a solution.

\section{b. Detachment of single-lobe meanders}

The single lobe meander is central to our discussion of the pinching off process. First consider the initial condition

$$
L(x, 0)= \begin{cases}\hat{L}_{2}\left\{1+\tanh \left[S_{0}(x+w)\right]\right\}, & x<0 \\ \hat{L}_{2}\left\{1-\tanh \left[S_{0}(x-w)\right]\right\}, & x>0\end{cases}
$$

consisting of a single ridge of amplitude $\hat{L}_{2}$, half-width $w$, and maximum slope $S_{0}$. Results for the case $\hat{L}_{2}=2$, $w=2, S_{0}=10$ are plotted in Fig. 10. At $t=0$ the disturbance resembles a "top hat" with rounded corners. Since the east and west faces of the top hat are four "deformation radii" apart, little communication initially exists between them. Therefore, the west face initially evolves as the step of Fig. 9, while the east face evolves as its mirror image (see $t=3$ of Fig. 10). As expected, vortex induction dominates the upstream face and causes it to unfold $(t=6)$ and disperse ( $t$ $=34)$. On the downstream face, however, remarkably steep slopes persist long after the upstream face has dispersed (see the segment ab at $t=34$ ). These steep slopes can be better understood by considering the north-south velocities produced by the vortex anomaly due to the ridge. Since this anomaly is anticyclonic, northward velocities are produced near the northwest corner of the ridge and southward velocities are produced near the southeast corner, as indicated by arrows in the $t=6$ profile of Fig. 10. Furthermore, the southward velocities produce a trough in the lee of the ridge, and the trough's cyclonic vortex anomaly tends to reinforce the already strong southward velocities between the ridge and crest. The downstream face of the crest is therefore caught between areas of strong northward excursions (upstream) and strong southward ex-

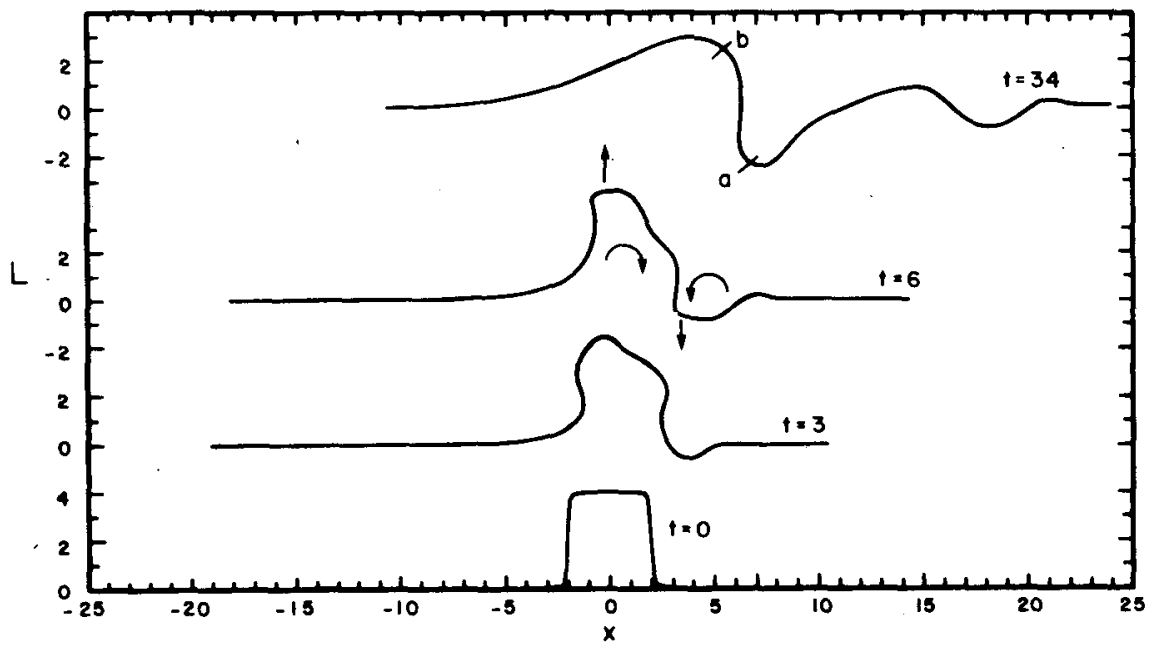

FIG. 10. Evolution of the case in which $L(x, 0)$ is given by Eq. (5.2) with $L_{2}=2, W=2, S_{0}=10$. 


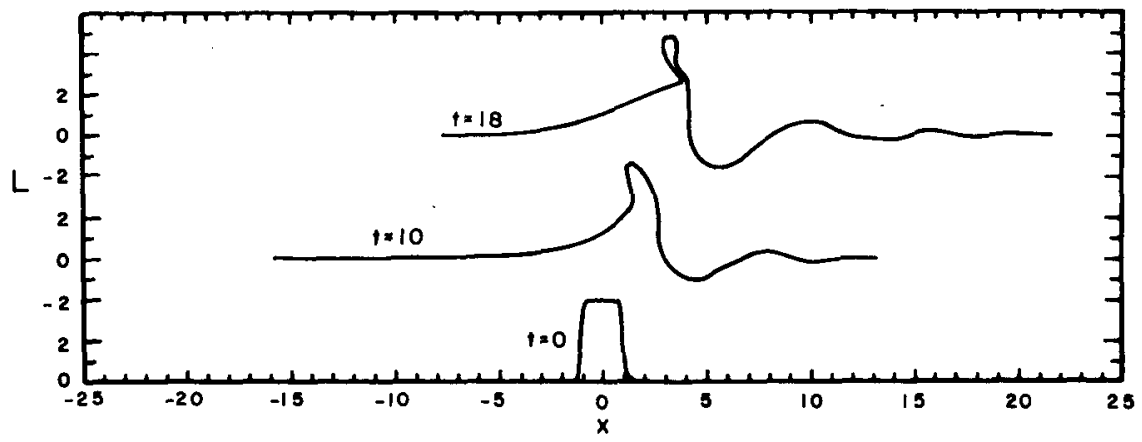

FiG. 11. As in Fig. 10 but $W=1$.

cursions (downstream), and this preserves the steep slope. Also, since the upstream face of the trough lies to the south of $y=0$, it will be steepened by the basic shear.

Although the ridge appears at $t=3$ to be on its way to pinching off at small $y$, this is prevented by dispersion at the upstream face. One way to prevent this dispersion, as suggested by section $4 \mathrm{~d}$, is to make the width of the initial disturbance smaller so that the basic field advection dominates and we therefore decreased the half-width to $w=1$. As shown in Fig. 11, the folding of the upstream face now continues unchecked until close contact is made with the downstream face. The latter maintains a steep slope despite the decreased strength of the initial vortex anomaly. At $t=18$ we see a blob beginning to detach, although the horizontal area of this blob $(\approx 0.64)$ is tiny compared to that of the initial area of the disturbance $(\approx 8.6)$. If $w$ is further reduced to 0.5 (Fig. 12), the bottom of the lobe is readily sheared off by the basic flow, and the detached area $(\approx 2.5)$ is now closer to the initial area $(\approx 4.9)$. In both cases note that the neck of the detaching blob is being stretched and tilted in a sense consistent with the mean shear.

These calculations suggest that fairly extreme initial conditions (i.e., small width and large amplitude) are required to allow single lobe disturbances to pinch off. This fact is further illustrated by considering an initial Gaussian profile.

$$
L(x, 0)=\tilde{A} e^{-(x / \tilde{w})^{2}}
$$

with $\tilde{A}=3$, and $\tilde{w}=1$. This profile has essentially the same width as the Fig. 11 initial profile but smaller amplitude ( 3 as opposed to 4 ) and a moderate maximum slope $\approx 1.8$. The numerical results obtained for this case (not shown) verify that the ridge fails to pinch off.

\section{c. Two-lobed meanders}

We now consider the case where the initial disturbance consists of a trough with positive vorticity anomaly lying downstream of a ridge with negative vorticity anomaly. (Note that the inverted case of a trough lying upstream can be obtained by merely replacing $L$ by $-L$ in that which follows.) In the previous examples, where we started with a single ridge, a cyclonic lobe was generated downstream as a result of vortex induction. In the present case introducing the trough $a b$ initio enhances the steepening that occurs on the downstream face of the ridge, and this can cause the ridge to pinch off before the upstream face has had a chance to disperse.

A simple and adequate initial distribution which will illustrate the point is

$$
L(x, 0)=-\hat{A} x e^{-(x / \hat{w})^{2}}
$$

and we note that the maximum amplitude of each of the two lobes is considerably less than nominal amplitude $\hat{A}$. The numerical results (Figs. 13 and 14) for $\hat{A}=6, \hat{w}=1$ (actual amplitude $=2.6$ ) show a small blob starting to detach from the ridge at $t=9$, and this

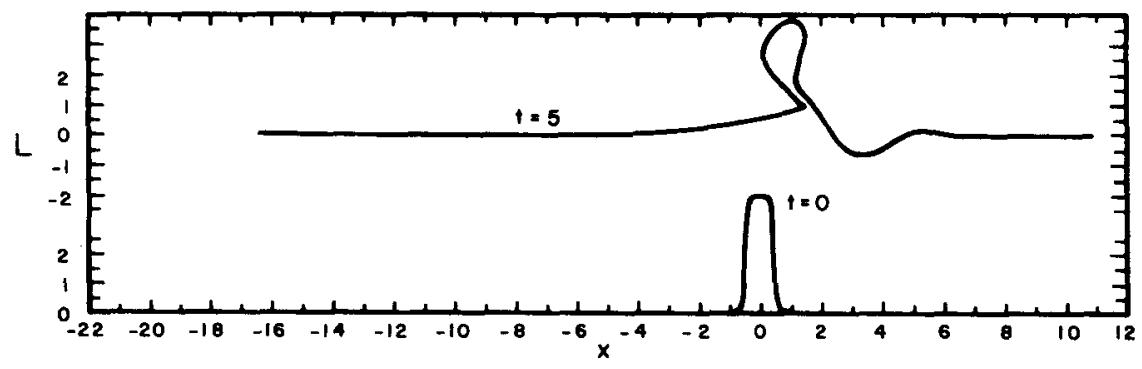

FIG. 12. As in Fig. 11 but $W=0.5$. 


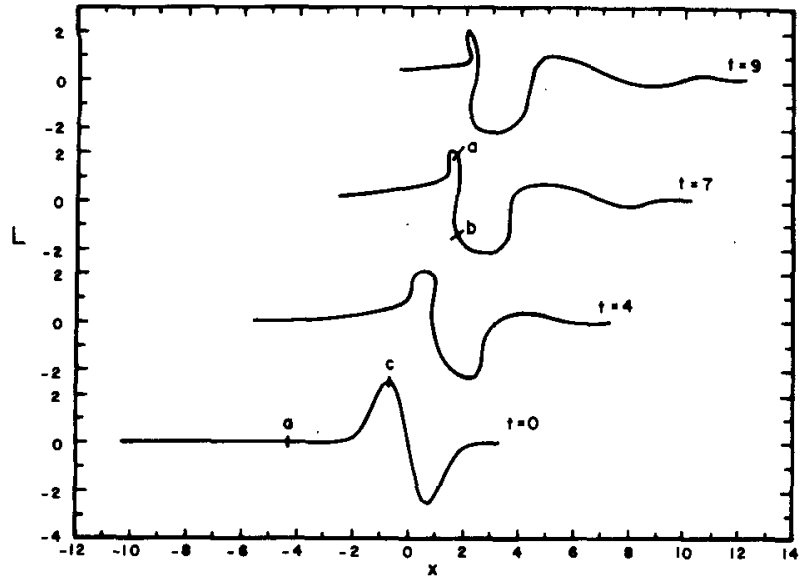

FiG. 13. Evolution of case $L(x, 0)=-\hat{A} x \exp \left[-(x / \hat{w})^{2}\right]$ with $\hat{A}=6, \hat{w}=1$. (Actual amplitude $=2.6$.)

result is to be compared with the previously described run 1d (Table I) where a single-lobed Gaussian of slightly larger $\max L(x, 0)$ and unit half-width failed to detach. Note the steepening of the front upstream of the trough along the segment labeled ab in Fig. 13 at $t=7$. Also note that the width of the trough grows while that of the ridge shrinks. As its area decreases, the ridge evolves more and more according to the spike model of section $4 \mathrm{~d}$. A small blob starts to pinch off $(t=11$, Fig. 14), but at the same time is stretched into a filament.

A detached eddy of significantly greater area and of ringlike appearance occurs when $\hat{A}$ is increased to 9.0, causing $\max |L(x, 0)|$ to increase by about 1.2 over the previous case. The early evolution (not shown) is similar to that discussed above and Fig. 15 shows a detailed view of the detaching eddy. Although its neck is being tilted and stretched in a way consistent with the basic shear effect, the eddy itself maintains an approximately elliptical shape. This integrity may be contrasted with stretching and shearing which dominates the smaller eddy of Fig. 14. The difference between the two cases is primarily due to the greater distance the eddy of Fig. 15 has from the region of strong basic shear (near $y$ $=0$ ). The center of mass $(\bar{y})$ of the latter eddy is approximately 2.4 , while $\bar{y} \approx 1.4$ for the eddy of Fig. 14 . Also the eddy of Fig. 15 is larger and therefore able to exert more influence on its surroundings, thereby resisting being passively advected by the basic flow.

\section{d. Three-lobed meanders}

We have seen that rather high initial amplitudes are required to inhibit the vortex-induced dispersion of the upstream face of a two-lobed disturbance and allow detachment. We now show that detachment can occur at much lower amplitudes if a small wavelet is incident from the west, so that the whole initial three-lobed system may be represented by

$$
L(x, 0)=-\hat{A} x e^{-(x / \hat{w})^{2}}-A_{n} e^{-\left[(x+D) / w_{n}\right]^{2}}
$$

where $A_{n}$ and $w_{n}$ denote the nominal amplitude and width of the new wavelet (lobe) and $D$ its offset. Although the two-lobe case $\hat{A}=6, \hat{w}=1.5, A_{n}=0$ produces no detached eddy (see run $2 \mathrm{a}$ of Table I), when we set $A_{n}=w_{n}=1$ and $D=2.5$ we do get a detached eddy as indicated in Fig. 16. The remarkable thing here is that the relatively small initial amplitude of the western wavelet $(\min L \approx-0.4)$ produces such a great qualitative change. Apparently the shorter wavelength and greater propagation speed of the wavelet prevents dispersion of the upstream face of the ridge. Our isolation of this effect may help to explain Ikeda and Apel's (1981) observations which show short eastward-propagating meanders catching up with longer meanders and apparently triggering eddy detachment.

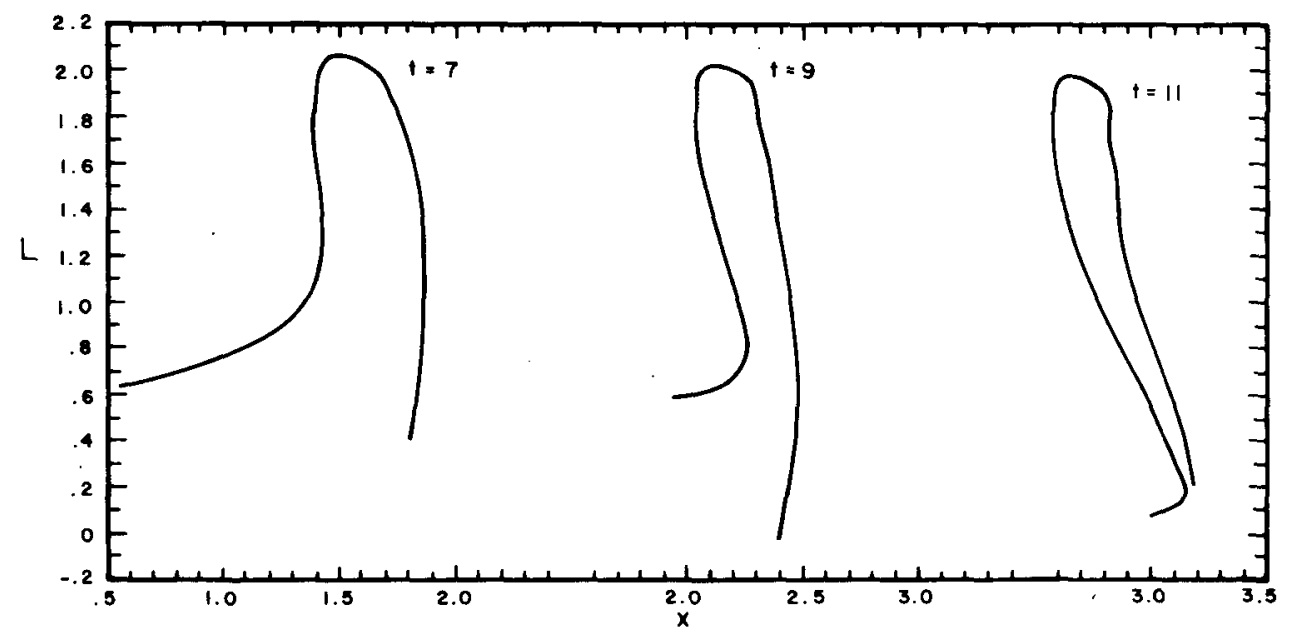

FIG. 14. Closeup of detaching blob in Fig. 13. The three profiles shown have been separated for visual convenience. 
TABLE 1. Summary of numerical experiments for free jet case.

\begin{tabular}{|c|c|c|c|c|c|c|}
\hline Run & $\begin{array}{l}\text { Initial } \\
\text { number } \\
\text { of lobes }\end{array}$ & Initial shape: $L(x, 0)$ & Figure & $\begin{array}{l}\text { Detached } \\
\text { area }\end{array}$ & $\frac{\text { Detached area* }}{\text { Initial area* }}$ & $\begin{array}{l}\text { Time of } \\
\text { detachment }\end{array}$ \\
\hline $0 \mathrm{a}$ & 0 & Eq. $(5.1), L_{2}=4 ., S_{0}=10$ & 9 & \multicolumn{2}{|c|}{ no detachment } & \\
\hline la & 1 & Eq. (5.2), $\hat{L}_{2}=2 ., S_{0}=10, w=2$ & 10 & & chment & \\
\hline lb & 1 & Same as la but $w=1$ & 11 & 0.6 & 0.07 & $t=16$ \\
\hline lc & $i$ & Same as la but $w=0.5$ & 12 & 2.5 & 0.51 & $t=7$ \\
\hline 1d & 1 & $3.0 \exp \left[-x^{2}\right]$ & - & \multicolumn{2}{|c|}{ no detachment } & \\
\hline $2 a$ & 2 & Eq. $(5.4), \hat{A}=6 . \hat{w}=1.5$ & - & \multicolumn{2}{|c|}{ no detachment } & \\
\hline $2 b$ & 2 & Same as $2 b$ but $\hat{w}=1$ & 13,14 & 0.2 & 0.08 & $t=10$ \\
\hline $2 c$ & 2 & Same as $2 \mathrm{~b}$ but $\hat{A}=9$ & 15 & 1.4 & 0.32 & $t=9$ \\
\hline $3 a$ & 3 & $\begin{array}{l}\text { Eq. }(5.5), \hat{A}=6, \hat{w}=1.5, D=2.5, \\
\quad A_{n}=3.86, w_{n}=1\end{array}$ & 17,18 & 4.0 & 1.12 & 15 \\
\hline $3 b$ & 3 & Same as 3 a but $A_{n}=2$ & - & 3.2 & 0.75 & 13 \\
\hline 3c & 3 & Same as 3 a but $A_{n}=1$. & 16 & 1.4 & 0.25 & 15 \\
\hline $3 d$ & 3 & Same as $3 \mathrm{a}$ but $w_{n}=1.5$. & - & 5.1 & 2.10 & $>12$ \\
\hline $3 e$ & 3 & Same as $3 \mathrm{~d}$ but $D=6$ & - & \multicolumn{2}{|c|}{ no detachment } & \\
\hline
\end{tabular}

* Initial area $=$ area above $y=0$ of lobe from which eddy detaches.

${ }^{\dagger}$ Detachment time $=$ time when neck width becomes $<0.1$.

Figure 17 illustrates the result of increasing the nominal amplitude $A_{n}$ of the new lobe to 3.86 . The pinching off of the centerlobe $(t=14)$ now occurs at

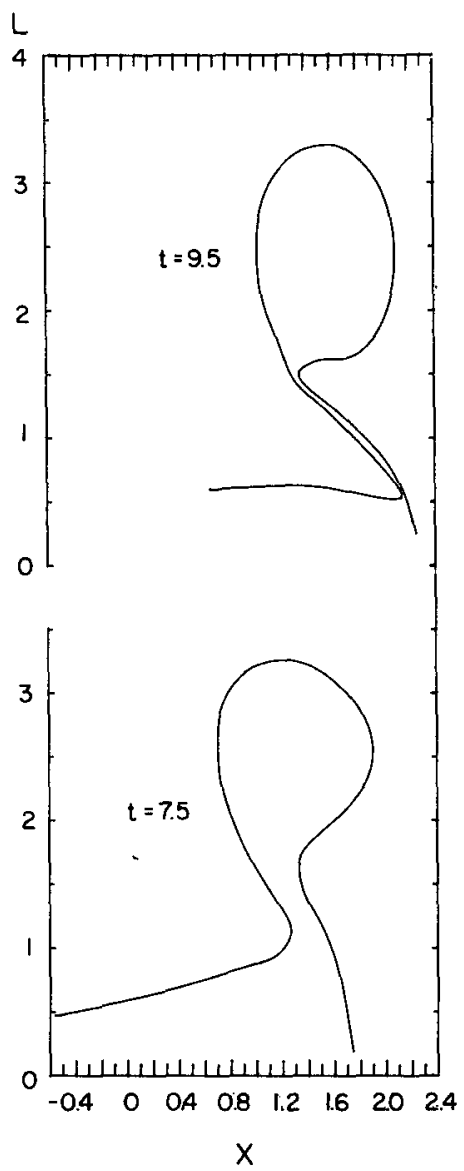

FIG. 15. Detailed view of the detaching eddy when the initial condition is the same as in Fig. 13 except $\hat{A}=9$. The profiles have been separated for visual convenience. a lower value of $y$, with the result that a greater area of fluid is trapped in the detaching eddy. The further evolution of the detaching eddy (Fig. 18) shows a tongue of high potential vorticity (cold) fluid wrapping around the southerly portion of the eddy. A similar tongue apparently occurs in the observations of Fig. 1. We note that tongues and necks such as those shown in Fig. 18 contain potential vorticity extremes and may therefore be subject to barotropic instability, although no firm evidence of the latter has been observed in any results.

Listed as a part of Table 1 are the results obtained using three-lobed initial profiles. Included are the horizontal areas of detaching eddies both in absolute terms and relative to the initial area (above $y=0$ ) of the lobe from which the eddy formed. In general, increases in the area (and vortex anomaly) of the west lobe tend to produce detached eddies of greater absolute and relative area. Taking run $3 \mathrm{a}$ as the benchmark case, we see that decreasing the nominal amplitude $A_{n}$ of the wavelet (runs $3 \mathrm{~b}$ and $3 \mathrm{c}$ ) results in a reduction of detached area. Increasing the nominal width $w_{n}$ of the west lobe increases the detached area as shown by run 3d, however if the displacement $D$ between west and center lobes is then increased from 2.5 to 6 (run $3 \mathrm{e}$ ) no detachment occurs.

We shall now try to bring these results together and suggest a generalized shape parameter $S$ which determines the relative area of detached fluid. The spike model discussed in section $4 \mathrm{~d}$ suggests that the initial aspect ratio (amplitude/width) of the detaching lobe should be of central importance. For large aspect ratios (i.e., narrow lobes), advection should overwhelm dispersion and large areas should detach. In Fig. 19 we have plotted relative detached area as a function of $S$ (= nominal amplitude/width scale) for all the one- and two-lobe runs. Clearly the detached area increases as $S$ increases, and there appears to be a critical value of 


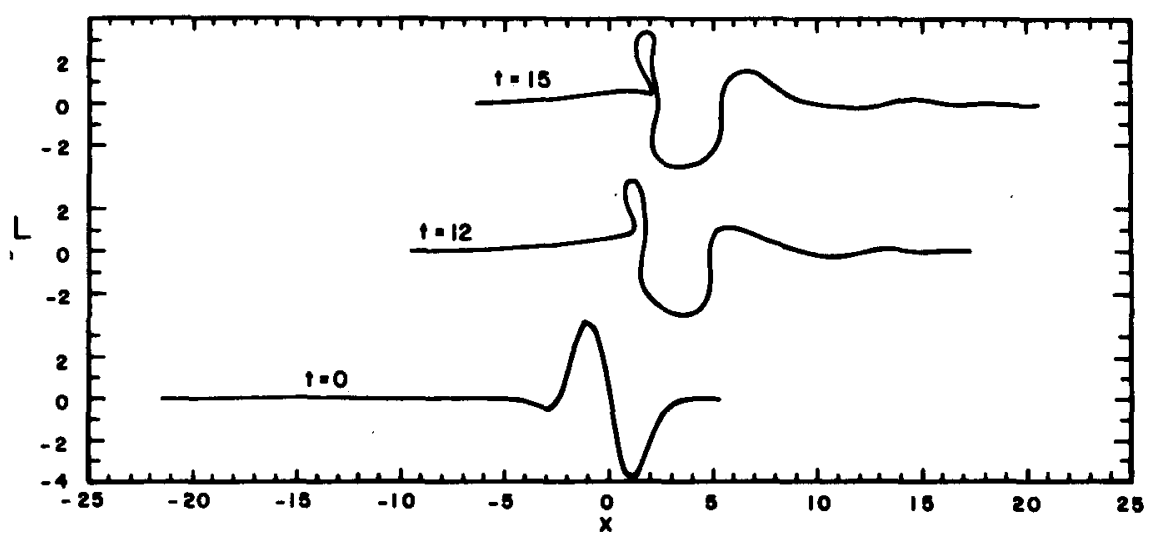

FIG. 16. Evolution of the case in which $L(x, 0)$ is given by (5.5) with $\hat{A}=6, \hat{w}=1.5, D=2.5$, and $A_{n}=w_{n}=1$.

$S$ (near 1.5) below which no detachment occurs. When three lobes are present, we have seen that the displacement $D$ and area $\left(-A_{n} w_{n}\right)$ of the west lobe strongly affect the detachment of the center lobe. There are a number of ways of constructing a shape parameter to include both the aspect ratio of the center lobe and the west lobe scales; here we choose to define $S=(\hat{A} / 2 \hat{w})$ $+A_{n} w_{n} / D^{2}$ (= center lobe aspect ratio + west lobe area/ square of displacement). A plot of relative detached area as a function of this generalized $S$ is shown in Fig. 19 , and the tendency of larger values of $S$ to produce larger detached areas is clear. There is also the suggestion that increasing the number of lobes allows milder initial shapes to produce large detached eddies. Further investigation of this sketchy relationship (Fig. 19) may provide a useful diagnostic tool for interpretation of satellite pictures of the Gulf Stream.

\section{e. A periodic case}

It is interesting and important to consider the evolution of a periodic disturbance such as

$$
L(x, 0)=A_{1} \sin \left(\pi x / w_{1}\right)-A_{3} \sin \left(3 \pi x / w_{1}\right)
$$

for the following reasons. First of all it is probably the simplest mathematical problem to pursue if one wants to obtain a deeper understanding of the detachment process. It has well-posed and rigorous (periodic) boundary conditions, as contrasted with the isolated disturbance for which it is necessary to assume no north-south velocity at the endpoints of the interval containing tagged parcels. Second, it would be desirable to extend the calculation to basic states which are unstable to small perturbations, e.g., three piecewise uniform potential vorticity regions (with a maximum in

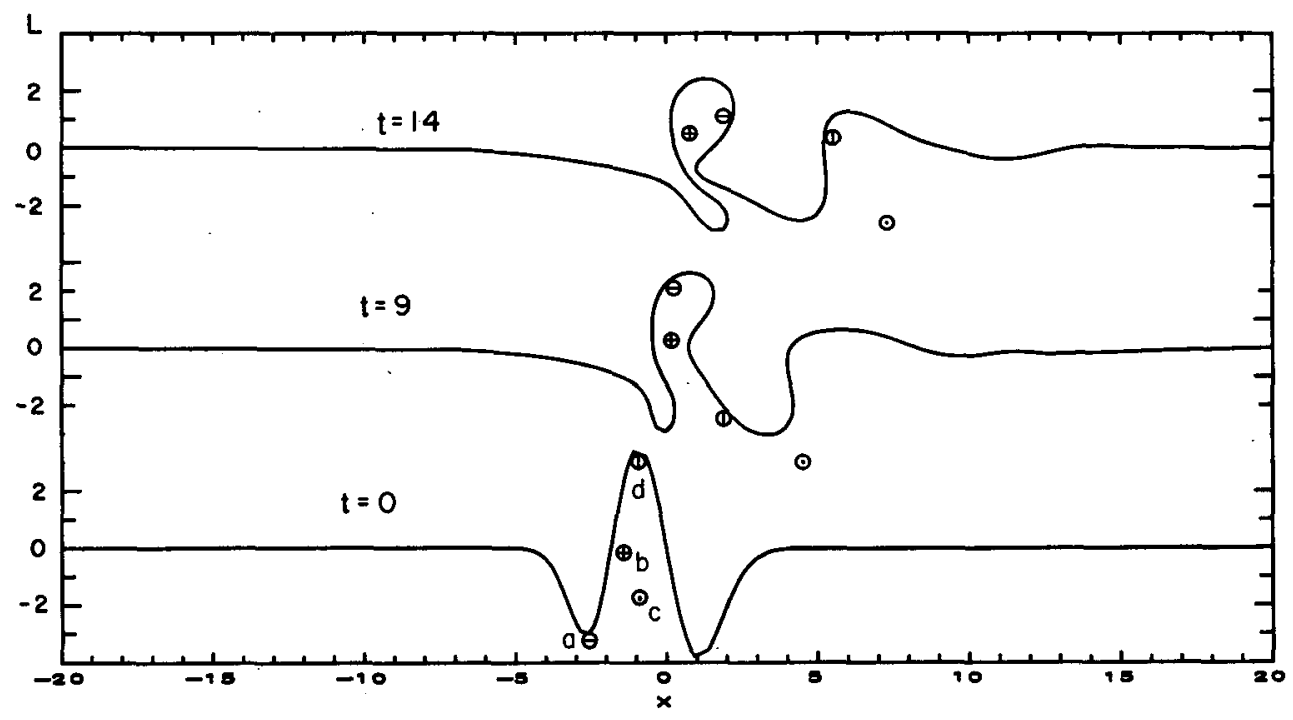

FIG. 17. As in Fig. 16 but with $A_{n}=3.86$. 


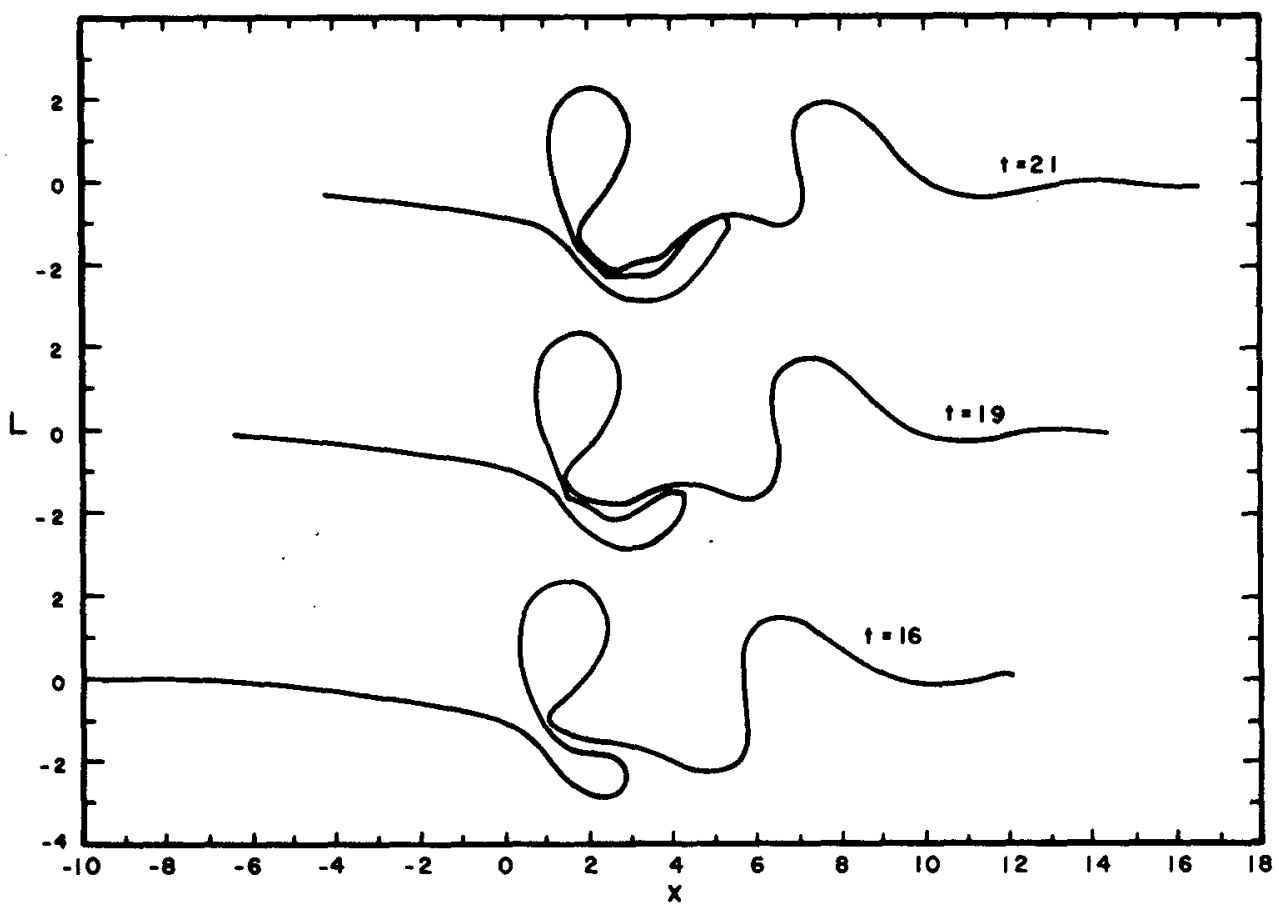

Fig. 18. Continuation of Fig. 17 to $t=21$.

the middle region) separated by two fronts. For this complex problem it is most straightforward to do the numerics using periodic disturbances, and the interpretations of the results will require a knowledge of the signatures produced in the absence of the instability mechanism. See the last part of the Appendix for the numerical method used in this calculation.

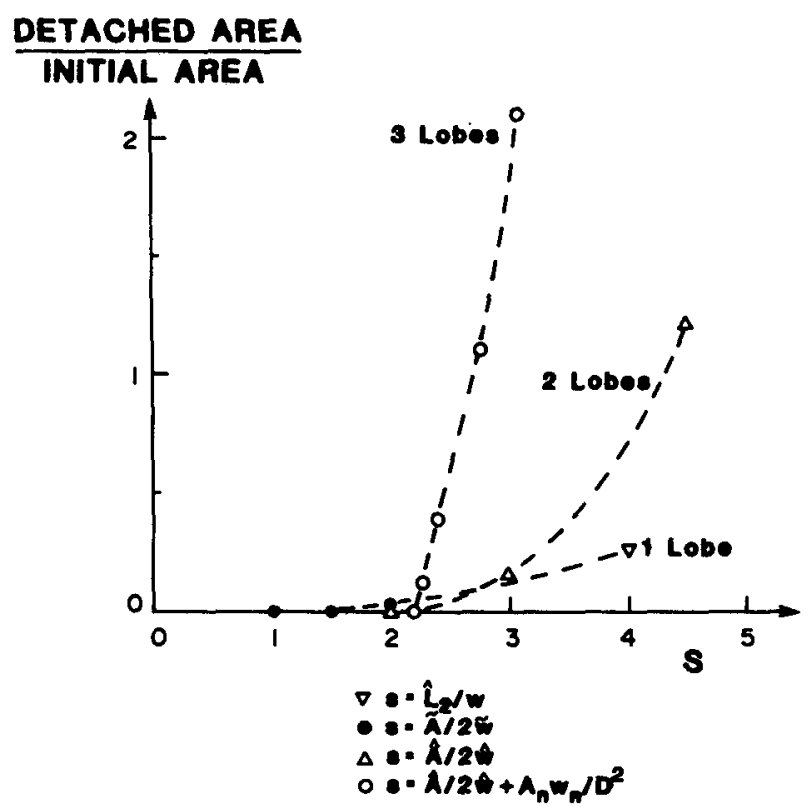

Fig. 19. Plot of relative detached area (as defined in Table 1) as a function of shape parameter $S$ for one-, two-, and three-lobe cases.
Figure 20a shows part of the evolution of a single $\left(A_{3}=0\right)$ sinusoid with $A_{1}=3.5$ and $w_{1}=1.5$. At $t=3, L(x, t)$ becomes multivalued as one side of each lobe steepens. Although the folding continues, no detachment occurs at later time (now shown) because the distance $\left(w_{1}\right)$ between zero crossings $(L=0)$ can be shown to be an invariant. Compare this with Fig. 20b for which the $w_{i}$ is the same, the fundamental amplitude is reduced to $A_{1}=1.75$, and a third harmonic of the same amplitude is added $\left(A_{3}=1.75\right)$. The cyclonic anomaly forming near $x=0$ at $t=3$ pinches off at about $t=6.3$ (not shown). Figure 21 shows the results when $A_{1}$ and $A_{3}$ are both halved. The pinched-off cyclone at $t=6.07$ shown in the upper left has been displaced for clarity from its proper position relative to the earlier curves. As was the case in section 5d, we see that the presence of the smaller wavelength allows a detached eddy to form.

\section{f. Velocities and parcel trajectories near a detaching eddy}

A disadvantage of the method of contour dynamics is that the streamfunction field is not given directly. Therefore it is difficult to obtain much of the diagnostic information that is readily available in finite difference simulations. However, the velocity field $[u(x, y, t)$, $v(x, y, t)]$ can be obtained using the contour dynamics algorithm by substituting $y$ for $L(x)$ in (3.3) and using the predetermined $L(\xi)$. (In terms of the algorithm presented in the Appendix, this amounts to substituting $y$ for $L_{i}$ and using the predetermined $L_{j}$.) 


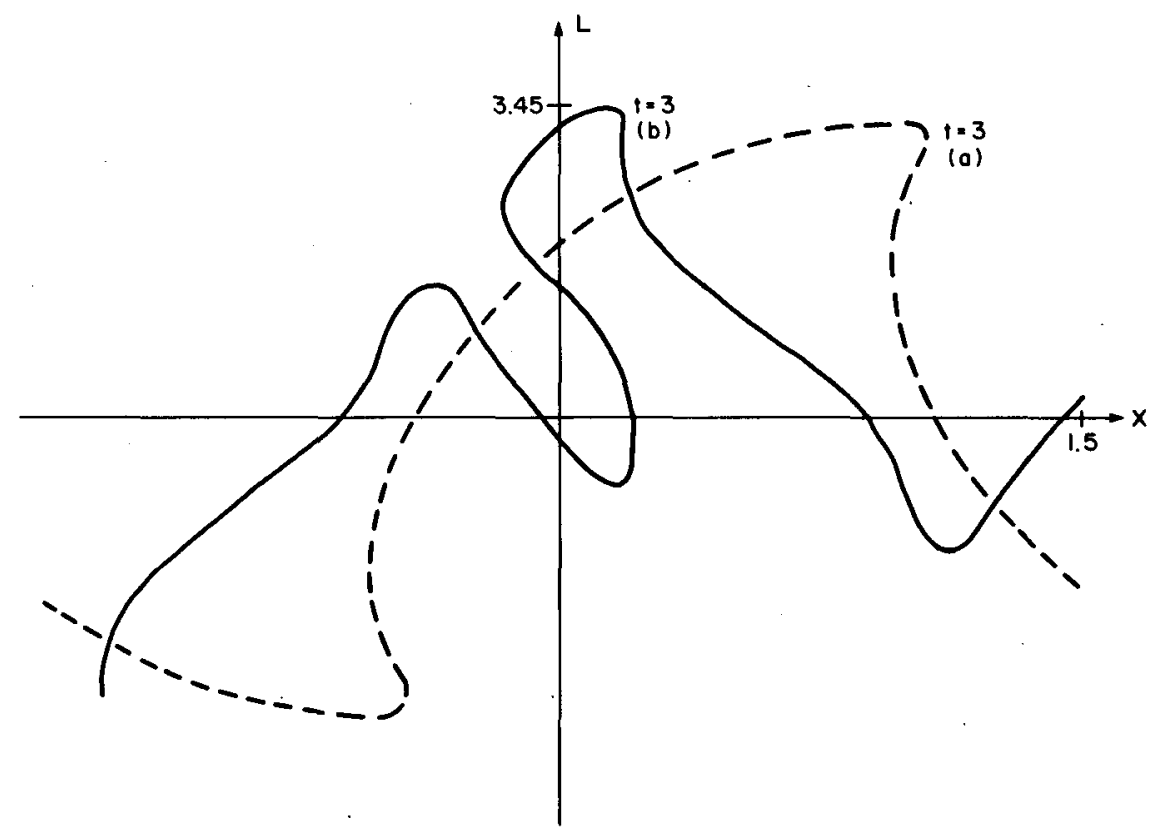

FIG. 20. Evolution of spatially periodic disturbances with the initial state given by equation (5.6). Curve a is the front at $t=3$ for the initial state $\left(A_{1}=3.5, A_{3}=0, w_{1}=1.5\right)$. Curve b shows the result when $A_{3}=A_{1}=1.75$.

As a typical case, we present (Fig. 22) the velocity field near the detaching ring of run $3 a$ (Figs. 17 and 18) at $t=14$. To the southwest of the neck, the tongue of high potential vorticity fluid can be seen moving southeast. Within the neck, the velocities are such as to close the neck and low potential vorticity fluid is being ejected in a southeasterly direction. Within the eddy, the circulation is anticyclonic as expected and the velocities normal to the front indicate an eastward propagation. If the normal velocities around the perimeter of the eddy are used to compute a propagation velocity, the eastward speed is 0.17 . (The basic flow has eastward speed 0.22 at the center of mass of the eddy.)

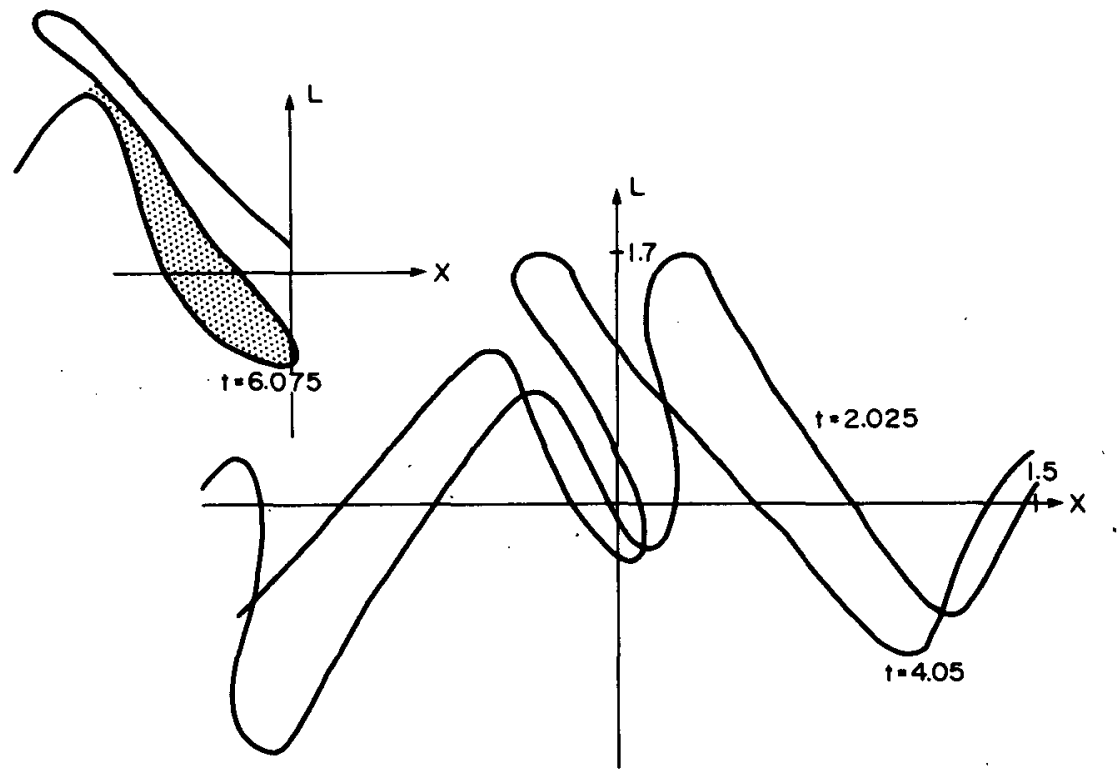

Fig. 21 . As in Fig. 20 but $A_{1}=A_{3}=0.875$. Inset shows pinching off cyclone. Although the amplitude is now smaller for the disturbance, more of it is influenced by the shear than in Fig. 19b. 


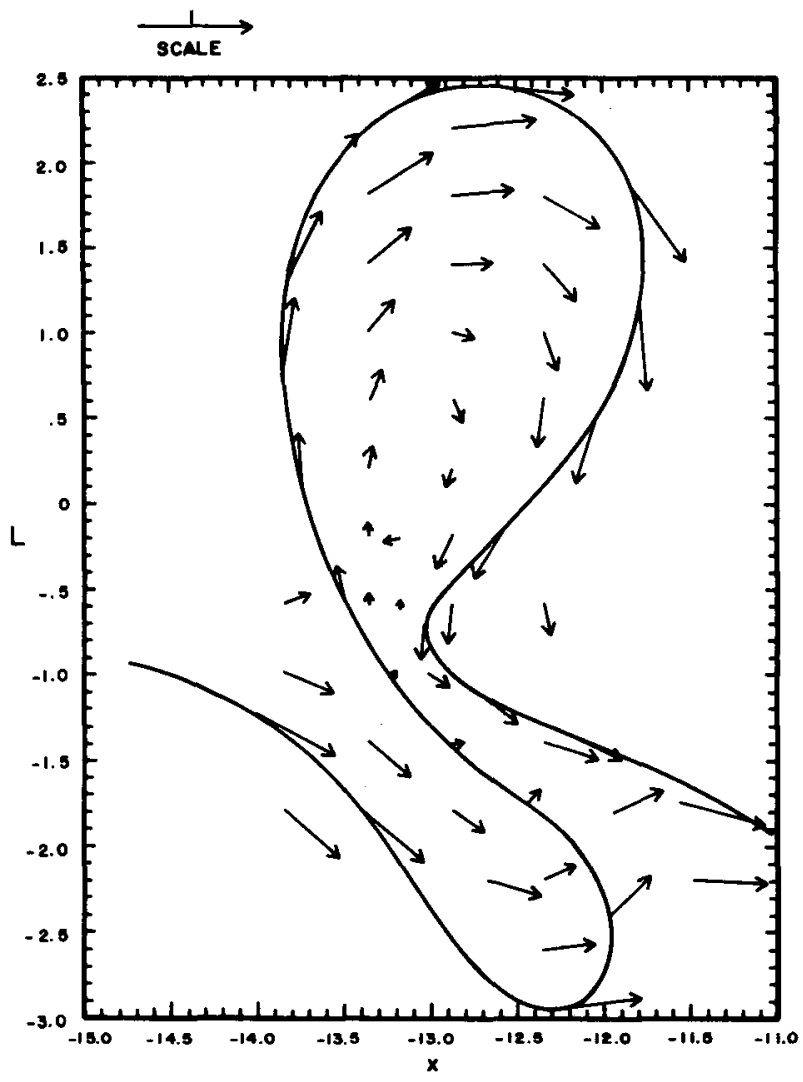

FIG. 22. Horizontal velocities near the detaching eddy of Fig. 17 at $t=14$.

The velocity fields for run $3 a$ have also been used to compute the trajectories of several tagged parcels near the eddy. The positions of these parcels are indicated by the small circles in Fig. 17 and it can be seen that all initially lie within the center lobe. Only parcels " $a$ " and " $b$ " are trapped within the detaching eddy; " $c$ " and " $d$ " are ejected and advected far to the east. This picture suggests that the detached eddy is composed primarily of fluid initially lying near the front to the west of the center lobe crest.

\section{An example with a lower boundary}

We have previously drawn attention to the rapid steepening which occurs on the downstream side of a meander (e.g., Fig. 13) and have partially attributed this to the strong north and south velocities produced by a positive feedback effect involving vortex induction. The strength of this important effect will now be demonstrated by showing that the rapid steepening and large velocities occur even when an east-west wall is inserted to block the path of the southward-moving parcels. Once again we set $B=0$ in $(3.1 \mathrm{a}, \mathrm{b})$ so that the boundary-intensified component of the basic flow is absent and the previously discussed frontal steepening tendencies are retained. Figure 23 shows the re- sult of a calculation in which $L(x, 0)$ consists of a Gaussian ridge [Eq. (5.3)] with $\tilde{A}=2, \tilde{w}=1$ and with $L_{0}=0.5$. The dotted curve shows part of the front at $t=6.8$ lying to the east of the meander crest, and the interesting part is to the west where the dots indicate positions of tagged parcels. We see that a steeply sloping wave front has formed near $x=-3.0$, and that the corresponding large southward velocities have led to grazing contact with the wall near $x=-2.6$. The curve with the arrows and the marked time points (open circles) shows the path taken by parcel 88 which contacts the wall at $t=6.8$. Figure 24 shows the result of similar calculations for different values of $L_{0}$ and $\tilde{A}$. The ground symbol indicates the time in the numerical calculation when grazing contact occurs with the wall, at which time the calculation is stopped. At this time the flow of the western branch of the current is blocked, and it is not at all clear how to continue the calculation in time. Increasing $L_{0}$ from $1 / 3$ to 0.75 with $\tilde{A}=2$ requires a longer time for blocking to occur, but contact with the wall still occurs despite the fact that the normal velocities must vanish there.

It is instructive to compare the above with a different kind of coastal current, for which the potential vorticity of the basic flow increases as one moves towards the wall, and thus we now set $A=0$ in Eq. (3.1a, b). We also choose $B=2$ so as to make the jump in potential vorticity across the front equal and opposite to that of the previously considered free jet. Figure 25 shows the shape of the front at time $t=6.0$ for $L_{0}=0.5$ and for a Gaussian initial bump having the same amplitude and width as for the case shown in Fig. 23. At earlier times the left-hand side of the disturbance folds rapidly and wave breaking occurs-all in a manner qualitatively similar to that which occurs when $L_{0}=\infty$ (Stern, 1985). The maximum downward displacement $(L=-0.455)$ of the front has already occurred at $t=4.5$ (not shown), and thus we see that the southward velocities in this case are insufficient to produce contact of the front and the coast.

\section{Summary}

We have shown how eddy detachment can occur from a simple cusped jet when no planetary potential vorticity gradient nor recirculating gyre exists. Most useful for this purpose and for future work on coastal jets is the contour dynamical equations for piecewise uniform potential vorticity currents. Also useful in providing a physical understanding of the numerical results is the simple formal decomposition of the latter equations into a basic flow component and a contribution due to vortex anomalies. For a northward displaced front (ridge) these anomalies are anticyclonic and are contained in the area between the disturbed front and the undisturbed jet axis. These anomalies will induce southward displacements of the front on the downstream side of the ridge (Fig. 10), and the 


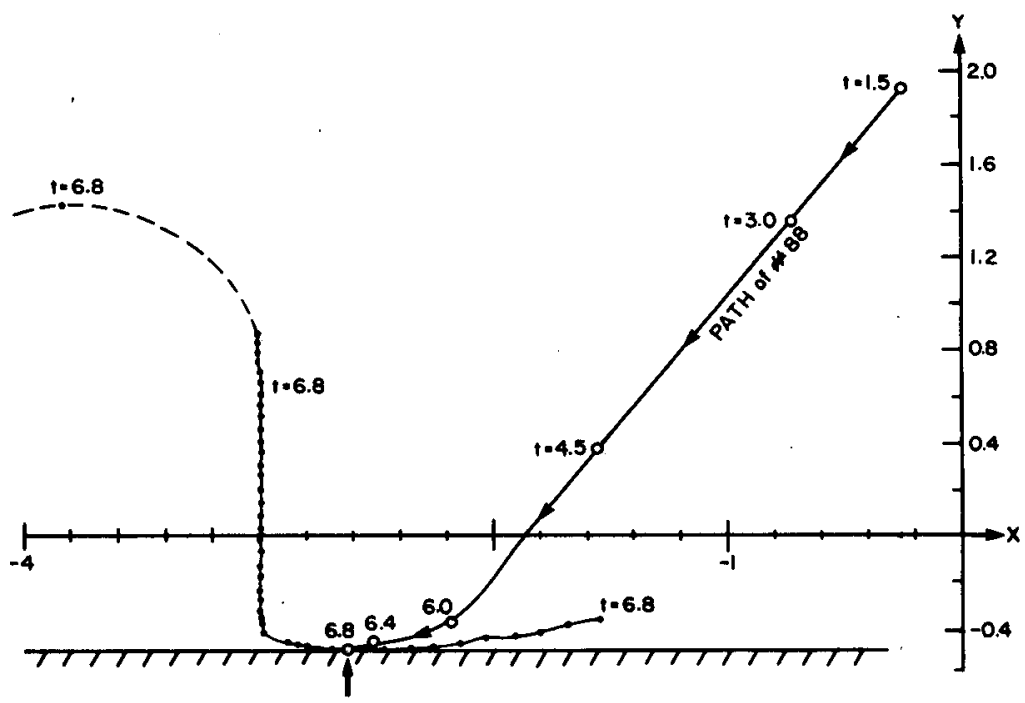

FIG. 23. Evolution for the case in which $L(x, 0)$ is a Gaussian (Eq. 5.3) with $\tilde{A}=2$, $\tilde{w}=1$ and a lower boundary is present at $L_{0}=0.5$. The small dots indicate the actual positions of a small portion of 125 tagged parcels along a section of the front which has made contact with the boundary at $t=6.8$. The arrowed curve connecting open circles shows the path taken by the first parcel (88) to contact the wall.

northward velocities on the other side are associated with pervasive dispersive effects tending to reduce the frontal slope on the upstream side of the ridge. This is not favorable for pinching off the whole ridge, whereas the southward velocities on the downstream face are favorable because they produce strong and persistent frontal steepening. Figure 23 illustrates the latter in a most impressive way because the coastal wall in that problem might be thought to inhibit the southward velocities and the concomittant steepening of the downstream side of the ridge. But in the case of the free jet this effect at the downstream face is not sufficient to produce a pinched-off ridge because the dispersion at the other face may prevent the two faces

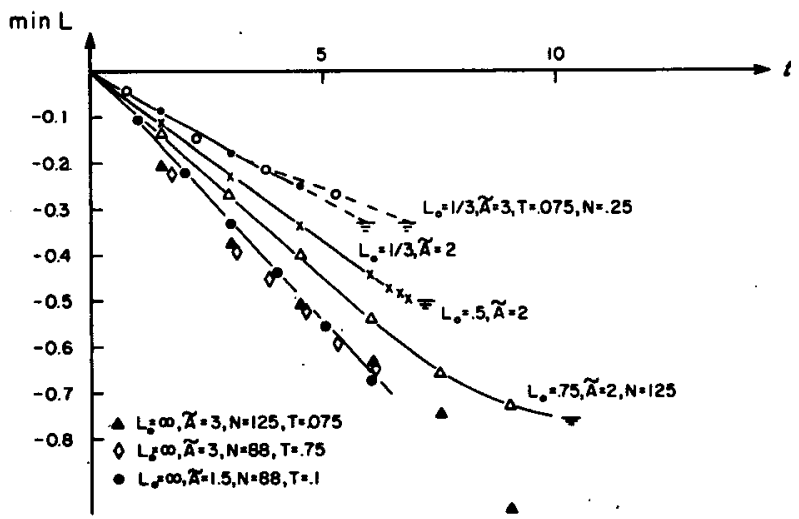

Fig. 24. Summary of calculations for cases similar to that of Fig. 23 but with different $L_{0}$ and $\tilde{A}$. The minimum $L$ is plotted against the time of its occurrence. $N$ is the number of Lagrangian points and $T$ is the time step. from joining up to form the stem (Fig. 15) precursor of the eddy detachment process. If the initial width of the ridge is sufficiently small, then this dispersion, associated with the vortex induction component, is small compared to the basic field advection component [Figs. $7,12]$, in which case the upstream face of the ridge steepens in time and joins the downstream edge in forming a detached eddy.

But relatively extreme amplitude and width conditions are required for this effect if the initial state consists of one or two lobes (Fig. 19). Smaller initial amplitudes are required when a small wavelet is incident on a ridge-trough meander of the main stream (Figs. 16-18). The wavelet offsets dispersion at the upstream side of the ridge, maintaining a steep slope there, and allowing it to catch up with the steep downstream slope of the ridge in question.

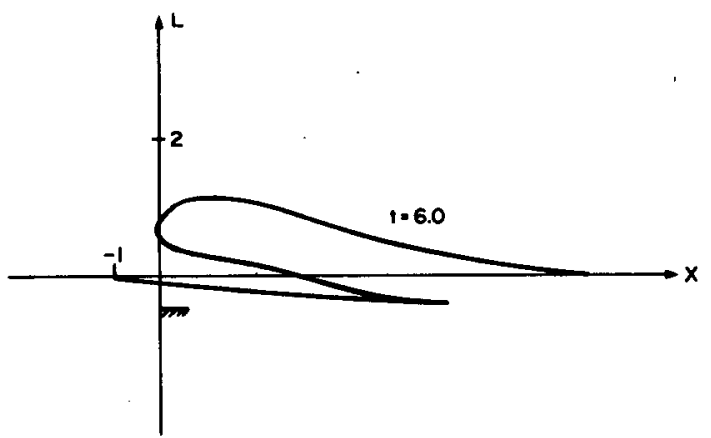

FIG. 25. The front at $t=6$ for the same initial state as Fig. 23 but with $(A=0, B=2)$. The minimum displacement $(L=-0.420)$ here does not reach the coast $y=-L_{0}=-0.50$. 
The later stages of Fig. 18 illustrate a limit of our calculation as well as the interesting close contact of the water masses at the converging neck of the disturbance. Further temporal integration would require a rapidly increasing computational resolution in the neck and a flagrant disregard of relevant nongeostrophic and smaller scale physical processes. At this point one is well advised to cut the cackle and the neck of the eddy in Fig. 18, continuing the quasi-geostrophic calculation with the resulting multi-connected domain, to see whether the eddy will remerge or be removed from the near vicinity of the Stream. Both of these effects appear to happen with observed warm core and cold core eddies in the Gulf Stream. Our results are applicable to the latter by merely changing the sign of $L$.

Acknowledgments. This work was supported by the Office of Naval Research under Contract N00014-81C-0062. The authors wish to thank Mrs. L. Nester for typing the manuscript.

\section{APPENDIX}

\section{Numerical Method}

The numerical procedure for solving (3.1) and (3.3 or 3.4) is based on a Lagrangian scheme in which $n$ tagged frontal parcels are followed. The position of any such parcel is denoted $\left[x_{i}(t), L_{i}(t)\right]$ where $i(=1, n)$ increases with increasing arclength along the front and where

$$
d x_{i} / d t=u\left(x_{i}, L_{i}\right) \quad d L_{i} / d t=v\left(x_{i}, L_{i}\right) .
$$

In the free case $\left(B=0, A=1, L_{0}=\infty\right)$, the $x$ velocity can be approximated from (3.3a) as follows

$$
\begin{aligned}
\pi u\left(x_{i}, L_{i}\right)= & \int_{-\infty}^{+\infty} d \xi K_{0}\left[R_{i}(\xi)\right] \\
= & \left\{\int_{-\infty}^{x_{1}}+\int_{x_{n}}^{\infty}\right\} d \xi K_{0}\left[R_{i}(\xi)\right]+\int_{x_{i-1}}^{x_{i+1}} d \xi K_{0} \\
& \times\left[R_{i}(\xi)\right]+\frac{1}{2} \sum_{\substack{j=2 \\
j \neq i, i+1}}^{n}\left(x_{j}-x_{j-1}\right) \\
& \times\left\{K_{0}\left[R_{i}\left(x_{j-1}\right)\right]+K_{0}\left[R_{i}\left(x_{j}\right)\right]\right\}
\end{aligned}
$$

where

$$
R_{i}(\xi)=\left[\left(x_{i}-\xi\right)^{2}+\left(L_{i}-L(\xi)\right)^{2}\right]^{1 / 2} .
$$

The three terms on the right-hand side of (A2) represent, in order, the contribution due to the portion of the front lying outside the interval $x_{1}<x<x_{n}$ containing tagged parcels, the contribution due to the integrable singularity at $x_{i}=\xi$ and the remaining contribution. The latter has been approximated using the trapezoidal rule. By employing a change in integration variable, the first term may be rewritten as

$$
\begin{aligned}
& \left\{\int_{-\infty}^{x_{1}}+\int_{x_{n}}^{\infty}\right\} d \xi K_{0}\left[R_{i}(\xi)\right] \\
& =\int_{R_{i}\left(x_{1}\right)}^{\infty} d \xi K_{0}(\xi) \xi /\left[\xi^{2}+\left(L_{i}-L_{1}\right)^{2}\right]^{1 / 2} \\
& \quad+\int_{R_{i}\left(x_{n}\right)}^{\infty} d \xi K_{0}(\xi) \xi /\left[\xi^{2}+\left(L_{i}-L_{n}\right)^{2}\right]^{1 / 2}
\end{aligned}
$$

Furthermore, we can write

$$
\begin{aligned}
& \xi /\left[\xi^{2}+\left(L_{i}-L_{1}\right)^{2}\right]^{1 / 2}=1+\mathrm{O}\left[\left(L_{i}-L_{1}\right) /\left(x_{i}-x_{1}\right)\right] \\
& \xi /\left[\xi^{2}+\left(L_{i}-L_{n}\right)^{2}\right]^{1 / 2}=1+\mathrm{O}\left[\left(L_{i}-L_{n}\right) /\left(x_{i}-x_{n}\right)\right] .
\end{aligned}
$$

If $x_{1}$ and $x_{n}$ are placed sufficiently far to the left and right of the isolated disturbance, then the value of each of the above expressions is nearly unity. Under these conditions we have

$$
\begin{aligned}
\left\{\int_{-\infty}^{x_{1}}+\int_{x_{n}}^{\infty}\right\} d \xi K_{0}\left[R_{i}(\xi)\right] & \\
& \approx\left\{\int_{-\infty}^{R_{i}\left(x_{1}\right)}+\int_{R_{i}\left(x_{n}\right)}^{\infty}\right\} d \xi K_{0}(\xi)
\end{aligned}
$$

Near the singularity $x_{i}=\xi, R_{i}(\xi) \ll 1$ and $K_{0}\left[R_{i}(\xi)\right]$ $\sim-\ln \left[R_{i}(\xi)\right]$. Therefore we may write

$$
\begin{gathered}
\int_{x_{i-1}}^{x_{i+1}} d \xi k_{0}\left[R_{i}(\xi)\right]=-\int d \xi \ln \left[R_{i}(\xi)\right]+\mathrm{O}(\Delta x) \\
\approx 1 / \pi\left\{2\left(x_{i+1}-x_{i-1}\right)-\left(x_{i+1}-x_{i}\right) \ln \left[\left(x_{i+1}-x_{i}\right)^{2}\right.\right. \\
\left.+\left(L_{i+1}-L_{i}\right)^{2}\right]-\left(x_{i}-x_{i-1}\right) \ln \left[\left(x_{i}-x_{i-1}\right)^{2}\right. \\
\left.\left.+\left(L_{i}-L_{i-1}\right)^{2}\right]\right\}
\end{gathered}
$$

where $\Delta x$ represents the spacing near the point $x_{i}=\xi$. The final step in (A4) has been performed through integration by parts and use of the trapezoidal rule.

From (3.3b) the $y$-velocity of the $i$ th parcel can be approximated as follows

$$
\begin{aligned}
\pi v\left(x_{i}, L_{i}\right)= & \int_{-\infty}^{+\infty} d L K_{0}\left[R_{i}(\xi)\right] \\
\approx & \int_{L_{i-1}}^{L_{i+1}} d L K_{0}\left[R_{i}(\xi)\right]+\frac{1}{2} \sum_{\substack{j=2 \\
j \neq i, i+1}}^{n}\left(L_{j}-L_{j-1}\right) \\
& \quad \times\left\{K_{0}\left[R_{i}\left(x_{j-1}\right)\right]+K_{0}\left[R_{i}\left(x_{j}\right)\right]\right\} .
\end{aligned}
$$

Here $x_{i}$ and $x_{n}$ are assumed to have been placed far enough away from the isolated disturbance so that $L$ $=$ constant for $x<x_{1}$ and $x>x_{n}$. Hence, no contribution to $v\left(x_{i}, L_{i}\right)$ arises from these intervals. The contribution due to the singularity (the first term on the right-hand side of A5) can be treated as in (A4) with the result 


$$
\begin{gathered}
\int_{L_{i-1}}^{L_{i+1}} d L K_{0}\left\{R_{i}(\xi)\right] \approx\left\{\left(L_{i+1}-L_{i-1}\right)-\frac{1}{2}\left(L_{i+1}-L_{i}\right)\right. \\
\times \ln \left[\left(x_{i+1}-x_{i}\right)^{2}+\left(L_{i+1}-L_{i}\right)^{2}\right]-\frac{1}{2}\left(L_{i}-L_{i-1}\right) \\
\left.\times \ln \left[\left(x_{i}-x_{i-1}\right)^{2}+\left(L_{i}-L_{i-1}\right)^{2}\right]\right\} .
\end{gathered}
$$

Equations (A2) and (A5) together with the approximations in (A3), (A4), and (A6) give the horizontal velocities for each tagged parcel. In most of the results presented here, 160 parcels are used to resolve the disturbance. Values of $x_{i}(0)$ and $L_{i}(0)$ are specified and a predictor-corrector scheme based on the Adams method (Gear, 1971) is used to integrate (A1).

During the time stepping, tagged parcels tend to clump together in regions of tangential velocity convergence and poorly resolved segments appear in divergent regions. It is therefore necessary to redistribute the parcels to maintain needed resolution, and this is done where necessary by reestablishing uniform spacing with respect to arc length. In order to perform integrations over much longer time intervals than those used here, more sophisticated redistribution schemes are required to handle the fine structure that develops. One such method is discussed by Zebusky and Overmann (1981).

A comparison was made between the numerical model and the exact linear solution for the initial state

$$
L(x, 0)=3 x /\left(x^{2}+4\right)^{2} .
$$

the amplitude $(\approx 0.12)$ and maximum steepness $(\approx 0.10)$ of which are small. The initial parcel spacing measured along the front is 0.1 and a relative timestepping error 0.0005 for each $\Delta t=1$ is specified. The maximum difference between the linear and computed value of $L(1.2, t)$ is $<0.5 \%$ over $0<t<10$.

A further test of the numerics is the ability to compute the horizontal and vertical velocities for the step shown in Fig. 8. For a uniform parcel spacing $=0.075$ along the front, the computed velocities are accurate within a relative error of 0.0015 .

The question arises as to the uniform validity of the quasi-geostrophic approximation when sharp corners in $L$ develop. Although it is easy to show that $(u, v)$ are finite, we would also like $(d u / d t, d v / d t)$ to be finite. Therefore we computed these quantities for the parcel located in the upper corner of the step profile (Fig. 8). As $t \rightarrow 0$ the acceleration of this parcel is bounded and of the same magnitude as the accelerations computed for neighboring parcels in the corner.

The extension to the wall-bounded jet (Fig. 22) is made by adding to (A2) and (A5) the terms due to the image vortices. These coastal calculations were made on an IBM PC, and follow quite closely the program used in Stern (1985). For the periodic case (Section Ve) the previous end point conditions are replaced by the sum of the periodic contributions outside of one wavelength $\left(=2 w_{1}\right)$. It can be shown from $(3.3 \mathrm{a}, \mathrm{b})$ that $L(x, t)=-L(x \pm w, t)$ at all $t$ if it is satisfied at $t=0$. Thus we only have to consider the Lagrangian evolution of points inside one half-wavelength $\left(w_{1}\right)$, taking into account the induction effect of all the other halfwavelength intervals. An adequate truncation was achieved by considering only four half-wavelengths on either side of the main half-wavelength. A second order Runge-Kutta time-stepping procedure was used in this program, and it was checked by setting $A_{3}=0, A_{1}$ $=0.005, w_{1}=2$, and by using $N=40$ Lagrangian points. The result gives phase speeds differing from linear theory by less than $1 \%$.

\section{REFERENCES}

Abramowitz, M., and I. A. Stegun, 1964: Handbook of Mathematical Functions. Dover, 1046 pp.

Flierl, G. R., and A. R. Robinson, 1984: On the time dependent meandering of a thin jet. J. Phys. Oceanogr., 14, 412-443.

Gear, C. W., 1971: Numerical Initial Value Problems in Ordinary Differential Equations. Prentice-Hall, $253 \mathrm{pp}$.

Hall, M. M., 1985: Horizontal and vertical structure of velocity, potential vorticity and energy in the Gulf Stream, Ph.D. thesis, MIT, 165 pp.

Ikeda, M., 1981: Meanders and detached eddies of a strong eastward flowing jet using a two-layer quasi-geostrophic model. J. Phys. Oceanogr., 11, 526-540.

Ikeda, M., and J. R. Apel, 1981: Mesoscale eddies detached from spatially growing meanders in an eastward-flowing oceanic jet using a two-layer quasi-geostrophic model. J. Phys. Oceanogr., 11, 1638-1661.

Magnus, W., and F. Oberhettinger, 1943: Formulas and Theorems for the Special Functions Mathematical Physics. Chelsea, 508 pp.

McWilliams, J. C., W. R. Holland and J. H. S. Chow, 1978: A description of numerical Antarctic Circumpolar Currents. Dyn. Atmos. Oceans, 2, 213-291.

Overman, E. A., and N. J. Zabusky, 1982: Evolution and merger of isolated vortex structures. Phys. Fluids, 25, 1297-1305.

Pullin, D. J., 1981: The nonlinear behavior of a constant vorticity layer at a wall. J. Fluid Mech., 108, 401-421.

Rhines, P. B., 1976: The dynamics of unsteady currents. The Sea: Ideas and Observations on Progress in the Study of the Seas, Vol. 6, Marine Modeling, E. D. Goldberg, I. N. McCave, J. J. O'Brien and J. H. Steele, Eds., Wiley-Interscience, 189-318.

Semtner, A. J., and Y. Mintz, 1977: Numerical simulation of the Gulf Stream and midocean eddies. J. Phys. Oceanogr., 7, 208230.

Stern, M. E., 1985: Large-scale lateral wave breaking and shingle formation. J. Phys. Oceanogr., 15, 1274-1283.

Stern, M. E., and L. J. Pratt, 1985: Dynamics of vorticity fronts. $J$. Fluid Mech. 161, 513-532.

Weatherly, G. L., and E. A. Kelley, 1985: Storms and Flow Reversals at the Hebble Site. Mar. Geol. 66, 205-218.

Weatherly, G. L., E. A. Kelley, M. Lopez and R. Harkema, 1984: The Gulf Stream system's frontal position and near bottom flow at the Hebble Site $\left(\sim 40^{\circ} \mathrm{N}, 62^{\circ} \mathrm{W}\right)$ from January 1982 to September 1982. Florida State Tech. Rep. 84-01, 63 pp.

Zabusky, N. J., and E. A. Overman, 1981: Regularization of contour dynamical algorithms. J. Comput. Phys., 52, 357.

-, M. Hughes and K. V. Roberts, 1979: Contour dynamics for the Euler equations in two dimensions. J. Comput. Phys., 30, 96-106. 